\title{
FUNCTIONALITY OF SOYBEAN CBF/DREB1 TRANSCRIPTION
}

FACTORS

Yuji Yamasaki ${ }^{\mathrm{a}, 1}$, Stephen K. Randall ${ }^{\mathrm{a},}$

${ }^{a}$ Biology Department, Indiana University - Purdue University Indianapolis

723 West Michigan Street, Indianapolis, IN 46202

Email addresses:

superexpress21@hotmail.com (Y. Yamasaki)

srandal@iupui.edu (S. Randall)

${ }^{1}$ Current address:

Department of Plant Sciences

222 Agriculture Building

66 Dafoe Road

University of Manitoba

Winnipeg, Manitoba

Canada

R3T 2N2

*Corresponding Author: Stephen Randall

srandal@iupui.edu 


\section{ABSTRACT}

Soybean (Glycine max) is considered to be cold intolerant and is not able to significantly acclimate to cold/freezing stress. In most cold tolerant plants, the Crepeat/DRE Binding Factors (CBF/DREBs) are critical contributors to successful cold-responses; rapidly increasing following cold treatment and regulating the induction of many cold responsive genes. In soybean vegetative tissue, we found strong, transient accumulation of CBF transcripts in response to cold stress; however, the soybean transcripts of typical cold responsive genes (homologues to Arabidopsis genes such as dehydrins, $A D H 1, R A P 2.1$, and LEA14), were not significantly altered. Soybean CBFs were found to be functional, as when expressed constitutively in Arabidopsis they increased the levels of AtCOR47 and AtRD29a transcripts and increased freezing tolerance as measured by a decrease in leaf freezing damage and ion leakage. Furthermore the constitutive expression of GmDREB1A;2 and GmDREB1B;1 in Arabidopsis led to stronger up-regulation of downstream genes and more freezing tolerance than $G m D R E B 1 A ; 1$, the gene whose transcript is the major contributor to total CBF/DREB1 transcripts in soybean. The inability for the soybean CBFs to significantly up regulate the soybean genes that contribute to cold tolerance is consistent with poor acclimation capability and the cold intolerance of soybean.

KEYWORDs: soybean; Glycine max; cold tolerance; CBF; DREB1

\section{ABBREVIATIONS:}




\section{INTRODUCTION}

Environmental stresses such as cold, drought, and salinity are serious problems for plants. Soybean is chilling and cold/freezing intolerant [1-4] with severe damage occuring at temperatures proximal to freezing. In the field, little growth occurs at temperatures below $6-7^{\circ} \mathrm{C}$ [5], and cool temperatures at the end of the growing season are a major limiting factor in soybean yield and production [6]. Unlike soybean, Arabidopsis thaliana is able to cold acclimate and thus survive under severe cold-stress conditions; e.g., -8 to 10 degrees Celsius [7, 8]. In Arabidopsis, the Crepeat response element binding factors (CBFs) play a key, though not exclusive role $[9,10]$, as positive regulators in an ABA-independent cold responsive pathway functioning to up-regulate many cold-regulated genes and thereby are critical contributors to cold/freezing tolerance [11]. CBF genes are members of the DREB (dehydration responsive element binding protein) family transcription factors which contain AP2 DNA-binding domains. AtCBF1, AtCBF2, and AtCBF3 (DREB1B, DREB1C, and DREB1A; respectively) are up-regulated significantly and rapidly by cold stress specifically, but much less so by ABA or dehydration [12, 13]. In contrast, AtCBF4 (DREB1D) and AtDREB2 genes are more specifically regulated by ABA, salinity, and dehydration but not by cold stress [12, 14, 15].

Both the DREB1 and DREB2 family of proteins have binding activity with elements containing the core nucleotide sequence CCGAC $[12,13,16]$. Genes up-regulated by constitutive expression of AtCBF3 (DREB1A) contain ACCGAC significantly more 
frequently than GCCGAC and TGTCGG in their promoters; while ACCGAC, CCGACT and GTCGGT are present in similar frequency in promoters of genes upregulated by AtDREB2A [17-19]_ENREF_17. Overexpression of AtDREB1A and AtDREB2A in Arabidopsis transgenic plants induce the expression of cold responsive genes such as $R D 29 a$ and $C O R 47$ without exposure to low temperature [20-22]. The accumulation of downstream genes mediated by $\mathrm{CBF} / D R E B 1$ genes, increase abiotic stress tolerances to freezing, dehydration, and high salt [14, 22-26]. In cold-intolerant tomato, the transgenic expression of AtCBF1 gene increased cold regulated genes [27] $[28,29]$ and the expression of AtCBF1 in tomato increases freezing tolerance [28-30]. However, overexpressing tomato CBF (LeCBF1) in tomato did not result in increased freezing tolerance. The genomic organization of the CBF genes is quite different in soybean. In Arabidopsis and tomato the CBF genes are located in a cluster (neighboring genes) on a single chromosome, while in soybean the four CBF homologs are on four different chromosomes. Soybean genes, SCOF-1 and GmDREB2 (Arabidopsis DREB2-like) are transcription factors that respond to cold stress and the transgenic expression enhances freezing tolerance of Arabidopsis plants [31, 32]. Transgenic potato plants expressing SCOF-1 also showed increased low temperature stress tolerance [33]. Despite the presence of SCOF-1 and CBF-like DREB1 transcription factors, soybean is cold-intolerant [7, 8, 34]; genes homologous to key Arabidopsis cold responsive genes, such as vegetative dehydrins and ADH, are not accumulated in response to cold stress [35, 36]. 
During the latter course of the described work, a comprehensive examination of the regulation of soybean CBF-like transcription factors by heat, cold, and drought stress, the functionality of several GmDREB1s in Arabidopsis and soybean, and the regulation by GmDREB1B;1 suggested the participation of DREB1 transcription factors in a variety of abiotic stress conditions [37]. The present report extends these findings by reporting on genes for GmCBF-like proteins (including the most highly constitutive and cold-induced expressed, CBF-like DREB1, GmDREB1A;1) that were not examined previously as well as providing a comprehensive RNASEQ analysis of cold regulated DREBs and the examination of the kinetics of CBF responses to cold. We further examined the responsiveness of soybean to cold by examining the kinetics of responsiveness of soybean CBF-like, DREB1 transcription factors, the functionality of these factors, and the changes in transcript levels of potential cold responsive genes in soybean. It was observed that several soybean CBF-like (GmDREB1) transcription factors can function to up-regulate characterized CBF responsive Arabidopsis genes and further can confer cold-tolerance when expressed transgenically in Arabidopsis. This paper supports a hypothesis that soybean lacks an appropriate transcriptional response to cold despite the fact that the cold sensing and initial portions of the CBF/DREB1 dependent cold signaling pathway are functional. 


\section{MATERIALS AND METHODS}

\subsection{Growth conditions}

Seeds for Glycine max, 'Young' (PI 508266) were generously provided by Tommy Carter (USDA-ARS, NC State University, Raleigh, NC). 'Young' was used for all experiments except RNASEQ analysis which used Williams 82 (PI518671). Seeds were soaked overnight in water at room temperature and then sown in pots $(16.5 \mathrm{~cm} \mathrm{x}$ $11 \mathrm{~cm}$ tall) containing a potting soil composed of a mixture of peat, perlite, and vermiculite (PRO-MIX BX, Premier Tech Horticulture) at a depth of approximately 2 $\mathrm{cm}$. Seedlings were grown and watered regularly in a plant growth chamber with $18 \mathrm{~h}$ light/6h dark at $22^{\circ} \mathrm{C}$. Lighting was 175 to $225 \mu$ mole $\mathrm{x} \mathrm{m}^{-2} \mathrm{x} \mathrm{sec}^{-1}$. Plants were not inoculated with rhizobium nor were they fertilized for these short-term experiments. Each replicate was created by combining plant organs from at least 4 plants (of similar developmental stage) from a minimum of 2 different pots. Cold treatment was for 2 days at $4^{\circ} \mathrm{C}$. Cold treatment $\left(4^{\circ} \mathrm{C}\right)$ was started at $4 \mathrm{~h}$ after the lights turned on (Zeitgeber Time, ZT4h) on day 10. Seedlings or unifoliate leaves and hypocotyls were harvested by freezing in liquid nitrogen followed by storage at $-80^{\circ} \mathrm{C}$ for later transcript analysis. Arabidopsis plants were grown under the same conditions as soybean and were typically used at four weeks old unless otherwise noted. For chemical rescue of growth phenotype, Arabidopsis transgenic plants grown on soil

were treated by spraying $10^{-4} \mathrm{M}$ of Gibberellin A3 (ACROS organics \#119860050) once a week starting with 10 day old seedlings until phenotype observation. 


\subsection{Creation of transgenic Arabidopsis plants expressing GmDREB1 genes}

The coding regions for GmDREBs were PCR amplified from Glycine max, 'Young' (PI 508266) cDNA with PfuUltra II Fusion HS DNA Polymerase (Agilent

Technology Cat. No. 600852) and primers were designed to add appropriate restriction enzyme sites for cloning (Table S1). The PCR products were cloned into pCR®-blunt (Invitrogen. Cat. No. K270020) and the sequence was confirmed. GmDREB1A;1, GmDREB1B;1, and GmDREB1B;2 sequences were identical to the sequence of the c.v. Williams 82 soybean sequence in the Phytozome site (Phytozome v9.1: http://www.phytozome.net/). GmDREB1A;2 had a single nucleotide difference ( $\mathrm{T}$ instead of $\mathrm{C}$ ) at 548 position resulting in the predicted amino acid methionine in “Young' rather than threonine found in "Williams 82". The coding region of GmDREB1 was cloned into pCambia1302 (replacing GFP) following the CaMV35S promoter. The Nos poly-A site of pCambia1302 was used as 3’UTR. The construct was transformed into Agrobacterium (GV3101) and then introduced into Arabidopsis (Col-2) WT plants by the floral dip method [38]. Hygromycin B resistant (15 $\mu \mathrm{g} / \mathrm{mL})$ seedlings were selected by a long hypocotyl phenotype [39], and the hygromycin resistant phenotype was calculated on the T2 generation and T3 generation to confirm presence of a single insertion and homozygous plants. Four-week-old transformed Arabidopsis seedlings were harvested by freezing in liquid nitrogen followed by storage at $-80^{\circ} \mathrm{C}$ for transcript analysis. 


\subsection{Transcript analysis}

For RT-qPCR, RNA from 100 mg of pulverized tissue (Arabidopsis

seedlings/soybean leaves or stems) in liquid nitrogen was obtained with the RNeasy

Plant Mini kit (Qiagen Cat. No. 74903). RNA treated with DNase (Qiagen Cat. No. 79254) during isolation, was then eluted from the column with water, and quantified

by absorbance at 260nm. Complementary DNA was synthesized with $500 \mathrm{ng}$ RNA, oligo dT primers and SuperScript ${ }^{\circledR}$ III First-Strand Synthesis System (Invitrogen. Cat. No. 18080051) in a $20 \mu \mathrm{L}$ reaction volume. Complementary DNA was diluted 4-fold ( $1 \mu \mathrm{L}$ represents $6.25 \mathrm{ng}$ RNA). One $\mu \mathrm{L}$ of 4 -fold diluted cDNA was analyzed with 400 or $500 \mathrm{nM}$ of each primer (Table S1) and $10 \mu \mathrm{L}$ Power SYBR® Green Master mix (Applied Biosystems ${ }^{\circledR}$ ) for a final $20 \mu \mathrm{L}$ total volume. Standard curves were created using the highest input cDNA ensuring all quantified values of samples were within the linear range. For absolute quantitation (absolute amount of cDNA), a PCR product was obtained by amplification from each respective gene, which was then purified and quantitated by UV absorbance and then used as template in PCR reactions to create linear standard curves (typically 10 attomoles to 40 femtomoles). This analysis allows direct comparison of the absolute levels of the distinct transcripts. All samples for quantification were in the linear range of the standard curve. PCR efficiencies were calculated and all data was within $85 \%$ to $105 \%$ efficiency $\left(\mathrm{R}^{2}>0.99\right)$. Dissociation curves were analyzed for all qPCR products and a single PCR product was confirmed. 


\subsection{RNASEQ analysis}

Unifoliate leaves from cold-treated soybean (c.v. Williams 82) seedlings (2 weeks old) were harvested at $0 \mathrm{~h}, 1 \mathrm{~h}$ and $24 \mathrm{~h}$. All treatments were performed in triplicate (with $\mathrm{n}$ $\geq 6$ plants per replication). RNA was isolated as described above and three libraries for each condition were created. RNASEQ analysis was performed by CGB genomics service facility, Indiana University (Bloomington, IN). Reads were mapped to the 68,552 transcript assembly using Bowtie 2.0 and then filtered to retain only the best alignments for each read. Reads were counted as the number of reads mapping to any transcript of that gene. Read counts were normalized across samples and adjusted Pvalue were obtained using the DESeq package (version 1.12) in R/Bioconductor. Normalized transcript count for each gene in the cold treatments were compared to control to analyze differential expression pattern.

\subsection{Analysis for whole plant freezing tolerance}

Potted Arabidopsis plants, four to five weeks old, were transferred to $-4.5^{\circ} \mathrm{C}$ for 24 hours in the dark followed by $4{ }^{\circ} \mathrm{C}$ for 24 hours in the dark and then returned to normal growth conditions for two days for recovery. The damage scale was scored after three days of the recovery, based on area and extent of damage on leaves (Figure S4).

\subsection{Electrolyte leakage}

Aerial portions of whole Arabidopsis plants, four to five weeks old, were harvested and transferred into $16 \times 100 \mathrm{~mm}$ glass test tubes. Plants were kept at $-1.0{ }^{\circ} \mathrm{C}$ for one 
hour, then an ice crystal was added. Plants were treated sequentially for two hours at each target temperature, then transferred into $4{ }^{\circ} \mathrm{C}$ in the dark for 12 hours. Treated plants were incubated with $3 \mathrm{~mL}$ deionized water for at least 6 hours with shaking. Conductivity was measured with a portable conductivity and TDS meter (Milwaukee Model MW301 EC meter). One hundred percent electrolyte leakage was determined following freezing of plants at $-80^{\circ} \mathrm{C}$ overnight.

\section{RESULTS}

\subsection{Identification of soybean CBF homologs}

Soybean DREB1 genes were found by searching for the presence of an AP2 DNA binding domain in the soybean genomic sequences (Phytozome v9.1: http://www.phytozome.net/). At least 44 DREB candidates (Figure 1) were found including those previously described such as GmDREB2 [32] and GmDREB2A;2 [26]. The most similar genes to Arabidopsis CBFs (AtDREB1s) were GmDREB1A;1, GmDREB1A;2, GmDREB1B;1, GmDREB1B;2, GmDREB1C;1, GmDREB1D;1 and GmDREB1D;2. In this group of CBF/DREB1-like genes, GmDREB1A; GmDREB1B;1, GmDREB1B;2 and GmDREB1D;1 were reported to be up-regulated at the transcript level in response to cold stress; the GmDREB1B;1 transcript additionally accumulated in response to dehydration [19]. While most of these genes exhibited strong responses to cold stress, they were also variably responsive to heat, salt and dehydration [37].. The GmDREB1A;1 homolog was reported to accumulate in response to dehydration stress in wild soybean [40]. All of these genes have very similar Nuclear Localization Signals (NLS) and AP2 domains followed by an acidic 
region at C-terminus. Unlike $C B F 1,2$ and 3 of Arabidopsis and tomato [13, 30], the soybean $C B F$ genes are not found as tandem arrays on the same chromosome (Chromosome 4 in Arabidopsis, Chromosome 3 in tomato), but rather were scattered among seven different soybean chromosomes. Soybean is a tetraploid plant, with about $75 \%$ of the genes having multiple copies [41]. Sequences of the gene pairs Glyma09g27180: Glyma16g32330, Glyma20g29410: Glyma10g38440 and Glyma17g14111: Glyma05g03560 are very similar to each other, suggesting duplication of these genes; while Glyma01g42500 does not have an apparent paralog.

\subsection{GmDREB1A;1 (Glyma09g27180) and GmDREB1A;2 (Glyma16g32330) are} strongly up-regulated in response to cold temperature.

Of the most closely related CBF-like genes, GmDREB1A;1, GmDREB1A;2, GmDREB1B;1 and GmDREB1B;2, only GmDREB1A;1 was not previously reported as a cold-inducible gene at 24 hours by micro array analysis [19]. However in Arabidopsis, AtCBF genes are strongly and transiently up-regulated; peaking at 2-4 hours following cold treatments and returning to much lower levels by 24 hours [42]. To determine whether cold-induced increases in the GmCBFDREB1 genes were transient, we examined transcript levels of GmDREB1A;1 and GmDREB1A;2 and a representative of another known cold-inducible transcription factor SCOF-1 (Glyma17g35430) [31] during early cold stress. The GmDREB1A;1 transcript was massively increased, up to 1000-fold, in leaves and stems following 3 hours of cold treatment (Figure 2). The transcript of the paralog gene, GmDREB1A;2, was also accumulated, but somewhat less so (Figure 2). Interestingly, while GmDREB1A;2 
was relatively more cold responsive in stem than in leaves, GmDREB1A;1 transcript was more strongly accumulated in leaves. Following strong accumulation in initial response to cold, both $G m D R E B 1 A ; 1$ and $G m D R E B 1 A ; 2$ were significantly decreased by 48 hours. These rapid, large and transient accumulations in response to cold stress are very similar to the transient expression pattern of Arabidopsis CBF genes [13]. While the level of the SCOF-1 transcript was also strongly increased in response to cold stress, the transient change (Figure 2) was much less than that observed in the DREB1A transcripts.

\subsection{GmCBF/DREB1 and $G m D R E B 2$ transcript changes in response to cold: RNASEQ analysis}

To determine the global transcriptional responses of soybean to cold, RNASEQ analysis was performed. We focus here on the analysis of the transcript level of primary transcription factors such as DREB and SCOF-1 related genes, and transcript levels of several soybean genes homologous to Arabidopsis cold responsive genes (Figure 3,4 and Figure S1). This experiment was designed to distinguish immediate responses (1 hour) and later responses (24 hour) to cold. In unifoliate leaves from young soybean seedlings, all of the GmCBF-like genes, [GmDREB1A;1 (Glyma09g27180), GmDREB1A;2 (Glyma16g32330), GmDREB1B;1 (Glyma20g29410), and GmDREB1B;2 (Glyma10g38440); as well as GmDREB1C;1 (Glyma01g42500), GmDREB1D;2 (Glyma17g14111), and GmDREB1D;1 (Glyma05g03560)] were significantly accumulated by 1 hour after cold treatment (adjusted P-value <0.001) (Figure 3). By 24 hours of cold treatment, transcripts of 
four GmCBF genes (GmDREB1A;1, GmDREB1A;2, GmDREB1C;1 and GmDREB1D;1) had significantly decreased compared to 1 hour but still sustained levels substantially above the 0 hour time control level (adjusted P-value <0.001). None of the soybean genes similar to AtDREB2 or 3 were up-regulated at 1 hour cold treatment, indicating that the $G m C B F / D R E B 1$ gene group are the immediate cold responsive genes acting as primary cold transcription factors (Figure 3). By 24 hours of cold treatment, transcripts of four DREB2-like genes (Glyma18g43750, Glyma14g06080, Glyma02g42960, and Glyma10g07756) were significantly accumulated, consistent with the previous observations of GmDREB2A;1 (Glyma02g42960) and GmDREB2A;2 (Glyma14g06080) cold responses [26]. It was interesting that the cold transcription factors, SCOF-1 (Glyma17g35430) and SCOF-1 like genes (Glyma06g04840, Glyma04g04760, Glyma20g26940, Glyma14g09760 and Glyma10g40400) were also significantly up-regulated within 1 hour of cold treatment and most sustained a significantly high level of transcript at 24 hours (Figure S1).

\subsection{Transcriptional changes of dehydrins and other potential cold responsive soybean genes.}

Dehydrins and alcohol dehydrogenase (ADH) are rapidly responding cold-inducible genes in Arabidopsis (e.g., AtCOR47 and AtADH1). Previously, of the ten dehydrin genes identified in the soybean genome; two KS-type dehydrins (Glyma17g24193 and Glyma16g04190) and GmERD14 (Glyma04g01130) were highly expressed in soybean leaves; while Mat9 (Glyma09g31740), Glyma08g05361, Glyma04g01181, Glyma12g36430 and a KS-type dehydrin (Glyma17g24193) were highly expressed in 
mature seeds [35]. Only the KS genes appeared cold-responsive, however; it was concluded that these genes responded primarily in a CBF/DREB1-independent (ABA-dependent) mode (Figure S2) as they lacked a putative CRT/DRE promoter element and they were responsive to exogenously applied ABA [35]. In the RNASEQ analysis (Figure 4), the two KS-type dehydrins (Glyma17g24193 and Glyma16g04190), as well as Mat9 (Glyma09g31740) and Mat1 (Glyma07g10030) were up-regulated by 24 hours in the cold (adjusted P-value <0.001); and the most abundant acidic dehydrin transcript in vegetative tissues under non-stressed conditions, GmERD14 (Glyma04g01130), was not up-regulated (Figure 4), consistent with the previous report by Yamasaki et al [35]. Soybean homologs of Arabidopsis cold responsive genes, GmADH10 (Glyma14g27940.1), AtRAP2 homolog (Glyma14g09320.1) and AtLEA14 homolog (Glyma09g38990.1) were examined (Figure S2). Despite the presence of putative abiotic stress responsive motifs in the promoters of GmRAP2 and GmLEA14, neither of these was significantly up-regulated.

\subsection{Regulation of Arabidopsis cold-responsive genes by GmCBF/DREB1s}

Cold-treated, wild-type Arabidopsis plants show a typical high level of accumulation of AtCBF3 expression by 1-4 hour of cold-stress, which then decreases to substantially lower levels by 24 hours (Figure S3). In response to CBF accumulation, Arabidopsis COR47, RD29a, and $A D H 1$ transcripts accumulated to their greatest level by 4 hours of cold stress (Figure 5), consistent with previous observations in Arabidopsis [23, 43]. Additionally, it has been shown, in the absence of any cold 
treatment, that the constitutive expression of AtCBF1-3 transcription factors increased the expression of AtRD29a, AtCOR47, and AtADH-1 [23, 43].

The responsiveness of these known Arabidopsis CBF regulated genes was utilized to evaluate the functionality of the soybean CBFs. Constructs containing GmDREB1A;1, GmDREB1A;2, GmDREB1B;1, or GmDREB1B;2 were introduced and constitutively expressed in Arabidopsis under the control of the cauliflower mosaic virus $35 S$ promoter (Figure 5). Non-transformed wild-type plants and homozygous transgenic plants expressing GmDREB1A;1 (3 lines), GmDREB1A;2 (2 lines), GmDREB1B;1 (2 lines), GmDREB1B;2 (2 lines) and GFP (1 line) were examined for the induction of several predicted responsive genes. Transgenic lines expressing high amounts of GmDREB1A;1 (09B-1-6, 09C-6-2), GmDREB1A;2 916A-4-5)or GmDREB1B;1 (20B-1-5) transcripts (Figure 6), showed strong accumulation of AtCOR47 and AtRD29a (Figure 5). It was interesting that only the overexpression line of $G m D R E B 1 B ; 1$ led to significant accumulation of AtADH1 transcript. Overall these data indicate that $G m D R E B 1 A ; 1,1 b$, and $2 a$ can function to up-regulate the Arabidopsis cold responsive genes, AtCOR47 and AtRD29a.

\subsection{GmCBF/DREB1 expression increases freezing stress tolerance.}

Non-acclimated wild-type and transgenic Arabidopsis plants treated at $-4.5^{\circ} \mathrm{C}$ for 24 hours were examined for damage. The extent of damage was estimated based on visible chlorophyll bleaching and leaf collapse (Figure S4). The wild-type plants and the transgenic plants expressing GFP were severely damaged, with component scale averages of 3.17 and 3.06, respectively (Table 1). The plants with high transcript 
levels of GmDREB1A;1, GmDREB1A;2, or GmDREB1B;1 were not damaged (component scale values of 0 to 0.1 ) indicating increased freezing tolerance of the plants (Table 1 and Figure 7). Two low level expresser lines of GmDREB1A;1( 09B2-2) and GmDREB1A;2 (16A-5-4) were heavily injured similar to wild-type line (Table 1). However, GmDREB1B;1 (20C-4-3), GmDREB1B;2 (10A-5-1 and 10A-4-1) had slightly less injury. To obtain a more quantitative evaluation of freezing tolerance, the high expression lines (09B-1-6, 09C-6-2, 16A-1-5 and 20B-1-5) were tested using the electrolyte leakage assay (Figure 8). All transgenic lines expressing high levels of soybean CBF/DREB1s showed significantly less leakage compared to wild-type and the GFP expression line. The lines of GmDREB1A;2 (16A-1-5) and GmDREB1B;1 (20B-1-5) were the most freezing tolerant based on the electrolyte leakage assay. These data indicate that the soybean GmDREB1A;1, GmDREB1A;2 and GmDREB1B;1 are able to confer freezing tolerance in Arabidopsis by transcriptionally activating CBF targets, as long as a sufficient level of GmCBF/DREB1 transcript is present.

\subsection{Transgenic expression of the GmCBF/DREB1 genes in Arabidopsis impacts the size of plants}

Arabidopsis plants over-expressing GmCBF/DREB1s (Figure 6) are dwarfed, a phenotype attributed to the suppression of the genes controlling plant growth and bolting [12, 24, 25]. To obtain a quantitative estimate of CBF/DREB1 transcript levels and to be able to quantitatively compare the levels of the distinct transcripts in different transgenic plants; the copy number of transcripts (contained in $6.25 \mathrm{ng}$ total 
RNA) for the GmDREB1 genes in the transgenic plants and non-transformed plants (4 weeks old) were absolute-quantified by Real-Time qPCR analysis (Figure 6 and Figure 6S). The two lines of GmDREB1A;1 (09B-1-6 and 09C-6-2), one line of GmDREB1A;2 (16A-1-5), and one line of GmDREB1B;1 (20B-1-5) all of which had the highest levels of the transcript ( $>10^{7}$ copies per 6.25 ng RNA) were dwarfed; while the lines expressing significantly less than $10^{7}$ copies, one line of GmDREB1A;1 (09B-2-2), one line of GmDREB1A;2 (16A-5-4), one line of GmDREB1B;1 (20C-4-3) and two lines of GmDREB1B;2 (10A-5-1 and 10A-4-1) appeared normal in size (Figure 6). Since the dwarfed phenotype of constitutively expressing AtCBF1 in tomato plants were complemented by application of GA3 [28]; we tested for the chemical rescue of the dwarf phenotype caused by GmCBFDREB1s. After six weeks of GA treatment, the inflorescence flowering structure of the dwarf plants, two lines of GmDREB1A;1 (09B-1-6 and 09C-6-2), one line of GmDREB1A;2 (16A-1-5), and one line of GmDREB1B;1 (20B-1-5) were partially complemented (size and branching, Figure S5). This suggested that the level of GmCBF/DREB1 expression in Arabidopsis affected GA biosynthesis or GA stability, leading to dwarfism. 


\section{Discussion}

\subsection{Comparison of cold-inducible $C B F / D R E B 1$ genes in soybean with those in}

\section{other plants}

Based upon protein sequence similarity of soybean DREB1 genes to Arabidopsis CBF genes; GmDREB1A;1, GmDREB1A;2, GmDREB1B;1, GmDREB1A;2, GmDREB1C;1, GmDREB1D;1, and GmDREB1D;2 (Glyma09g27180, Glyma16g32330, Glyma20g29410, Glyma10g38440, Glyma01g42500, Glyma05g03560 and Glyma17g14111 respectively) were hypothesized to be the most likely cold inducible soybean CBF-like genes (Figure 1). It is shown here that this hypothesis was clearly supported as these CBF-like transcripts accumulated rapidly, strongly, but transiently in response to cold in soybean. Further, several of these soybean CBF-like genes, when transgenically expressed, were capable to up-regulate typical downstream genes leading to the acquisition of cold tolerance in Arabidopsis. In contrast, none of the remaining DREB1 genes nor any of the twenty DREB2 genes responded after 1 hour of cold and only two increased significantly by 24 hours, with six decreasing in response to cold. Consistent with these observations, some members of the DREB2 family, both in Arabidopsis [12] and in soybean_ENREF_27 [26], are more strongly up-regulated in response to dehydration and salt stress than cold stress.

The Arabidopsis CBF 1-3 genes are closely-linked, tandemly arranged genes located on chromosome 4 [13] and likewise the CBF1-3 genes of tomato (S. lycopersicum, S. pimpinellifolium and S. habrochaites) and potato (S. tuberosum and S. commersonii) are also tandem-linked genes. In potato (S. tuberosum and $S$. 
commersonii), an additional set of tandem-linked CBF genes (CBF4-5) were found as orthologs to S. tuberosum and S. commersonii CBF1-3 [44]. Unlike the Arabidopsis, tomato, and potato CBF genes, the soybean $G m C B F$ genes are distributed on distinct chromosomes. The protein sequences of all GmCBF-like/DREB1 genes are most similar to that of AtCBF4 (AtDREB1d) sequence (Figure 1). AtCBF4 is thought to be derived from same ancestor as AtCBF1-3 based on coding sequence and promoter sequence similarity between AtCBF4 and AtCBF1-3 [14]. There is a greater degree of synteny in the neighboring genes to GmDREB1A and $1 B$ genes to the region surrounding AtCBF4 than to the region surrounding the tandem-linked AtCBF1-3 (data not shown); suggesting that the $G m C B F / D R E B 1$ genes may be derived more recently from the same ancestor as AtCBF4. Since soybean is thought to have duplicated its genome twice, at about 59 and 13 million years ago [41], we suggest that the genes pairs of GmDREB1A;1-GmDREB1A;2, GmDREB1;1-GmDREB1B;2, and GmDREB1D;1-GmDREB1D;2 may have originated through a series of duplications from one ancient $C B F 4$-like gene.

\subsection{GmCBF-like DREB1s as primary cold responsive transcription factors}

GmDREB1A;1 (Glyma09g27180) transcripts were accumulated from 400 to greater than 1,000-fold in leaves and 250 to 400 -fold in stems (Figure 2 and Figure 3). GmDREB1A;2 (Glyma16g32330) transcript was accumulated 10 to 40 -fold and 30 to 140-fold in leaves and stems, respectively after 1 hour of cold treatment (Figure 2). These responses are comparable to those found in Arabidopsis, where AtCBF3 transcript was up-regulated 300-fold within 1 hour of cold treatment (Figure S3). In 
Arabidopsis, CBF1-3 are the primary cold inducible transcription factors [12, 13], with AtCBF3 and AtCBF1-2 being first transcriptionally activated by ICE-1 and CAMTA, respectively [45-47]. There are five potential elements (myc-recognition sites, CANNTG) present in the promoter ( 1kb from the ATG) of AtCBF3 $[45,48]$ to which ICE1 could bind. The promoter of GmDREB1A;1 (Glyma09g27180) gene similarly has 2 and 9 Myc-recognition sites within $1 \mathrm{kbp}$ and $1.5 \mathrm{kbp}$, respectively while GmDREB1A;2 (Glyma16g32330) gene has 3 and 4 Myc-recognition sites in $1 \mathrm{kbp}$ and 1.5kbp; respectively. These potential ICE responsive elements are consistent with the hypothesis that soybean and Arabidopsis share initial steps in their cold-signaling mechanisms.

All of the seven genes encoding GmCBF/DREB1 proteins are up-regulated after 1 hour cold (Figure 3), while none of the GmDREB2 or 3 genes were significantly upregulated at 1 hour. These suggest the entire up-stream portion of the soybean CBF/DREB1 cold signaling pathway, including ICE1 and CAMTA, is functional to induce the expression of GmCBF/DREB1 genes leading to transcript accumulation. However, this activation is not sufficient to induce cold tolerance in soybean plants. In Arabidopsis, the accumulation of $C B F$ causes up-regulation of a downstream regulon $[19,23,25,49,50]$. In Arabidopsis the CRT/DRE is the most common element in cold inducible promoters; however, in soybean the most common element in the promoters of cold-induced genes are the ABRE and T/G box Maruyama et al. (2012). This further suggests that the soybean cold responsive pathway utilizing CBF is not functioning in the same way as in Arabidopsis. Never-the-less, in at least one 
case it has been demonstrated that GmDREB1B;1 can directly interact with the promoter of a stress responsive soybean promoter [37].

\subsection{Functionality of $G m C B F / D R E B 1$ genes depends on sufficient level of expression}

AtCBF1-3 accumulation in response to cold stress, induces the expression of the CBF regulon by binding CRT/DRE elements (A/GCCGAC) in the cold-responsive promoters [51]. Typically, overexpression of AtCBF1-3 increases the expression of AtRD29a and AtCOR47, utilizing 4 and 3 CRT/DRE elements present within $1 \mathrm{~kb}$ upstream of the ATG, respectively [23, 43]. Arabidopsis plants overexpressing AtCBF1-3, have a dwarf phenotype due to regulation of GA biosynthesis pathway by CBF genes [24, 25, 52]. Exogenous GA3 application returns the normal growth habit to transgenic tomato plants expressing AtCBF1 [28]. Here a range of constitutive levels of $G m C B F / D R E B 1$ transcripts in transgenic Arabidopsis were obtained (Figure 6 and Figure S5). A GmDREB1 expression difference of approximately ten-fold (Figure S6) seems sufficient to induce dwarfism (Figure 6 and Figure7), to determine the on or off status of cold-regulated genes (Figure 5 and Figure S5), and to determine freezing or non-freezing tolerance (Figure 7 and Figure 8). 


\subsection{GmDREB1B;1 has a regulatory pattern distinctive from $G m D R E B 1 A ; 1$ and}

\section{GmDREB1A;2.}

The downstream activated gene targets of GmDREB1B;1 have been carefully characterized [37]. When compared on a limited scale here, with other CBF-like DREB1's it was observed that the line with the highest level of GmDREB1B;1 (20B1-5) transcript, showed an increase of AtADH1 transcript greater than 3-fold; while the GmDREB1A;1 (09B-1-6 and 09C-6-2) and GmDREB1A;2 (16A-1-5) had less than a 2-fold increase (Figure 5 and S5). AtADH1 was reported to be a cold, dehydration, hypoxia, and ABA-inducible gene $[53,54]$. Regulation of the $A D H 1$ gene in Arabidopsis by AtCBF2 is suggested by experiments which alter accumulation of CBF2 [55-57] and overexpression of CBF2 [58]. Maruyama et al [19] _ENREF_19suggested the specific promoter elements regulated by DREB2 are ACCGAC, GTCGGT and CCGACT, while DREB1A/CBF3 regulated primarily ACCGAC. In the Arabidopsis ADH promoter, a GTCGGT sequence is found rather than the ACCGAC sequence. Perhaps in transgenic Arabidopsis plants, GmDREB1B;1 may better recognize the GTCGGT element (in AtADH) and thereby induce the expression of AtADH1.

\subsection{GmDREB1A;2 and GmDREB1B;1 expressing Arabidopsis plants have more freezing tolerance than those expressing GmDREB1A;1. \\ Based on electrolyte leakage, the constitutive GmDREB1A;2 and GmDREB1B;1 expression lines (16A-1-5 and 20B-1-5, respectively) were more freezing tolerant than GmDREB1A;1 lines (09B-1-6 and 09C-6-2), , even though all of the lines}


showed similar levels of accumulation of AtCOR47 and AtRD29a (Figure 5, Figure S5, and Figure 6). This may mean that distinctive promoter specification, perhaps impacting distinct genes regulated by GmDREB1A;2 or GmDREB1B;1 but not by GmDREB1A;1, results in more freezing tolerance.

\subsection{Soybean dehydrins are not directly regulated by the CBF pathway during cold stress}

The RNASEQ analysis indicated the KS-type dehydrins, Mat9 (Glyma09g31740), and Mat1 (Glyma07g10030) transcripts were accumulated in response to cold stress, but the rest of the dehydrins were not (Figure 4). It is likely the dehydrin genes are not directly regulated by the CBF-like DREB1s. The KS-type dehydrin, Glyma17g24193 contains ABRE sites (and no CRT/DRE), and is up-regulated by exogenous ABA treatment [35]. Mat9 (Glyma09g31740) and Mat1 (Glyma07g10030) are dehydrins which are primarily expressed in seeds and contain many potential ABRE elements [59, 60]. Maruyama et al [19]_ENREF_19showed a high frequency of ABREs in the promoters of cold inducible genes in soybean. This indirect regulation of dehydrin genes by cold is consistent with the demonstration of GmDREB1B:1 activation of ABRE-mediated gene expression [37]. It is likely that soybean has a robust ABA-dependent, cold response.

\subsection{SUMMARY}

Overall these findings confirm the initial steps in the CBF/DREB1 pathway in soybean are responsive to cold stress culminating in the accumulation of transcripts of 
$G m C B F / D R E B 1$ genes. Since the cold signal transduction pathway in soybean is appropriately functional, from the perception of cold to the elevation of GmCBF/DREB1 transcripts; the downstream components may be deficient in their cold response. Possible contributions to the lack of an appropriate cold response may include insufficient levels of $G m C B F / D R E B 1$ transcripts and/or protein, that are insufficiently accumulated to up-regulate downstream genes, absence of co-activators or presence of strong negatively acting transcriptional regulators, or the lack of appropriate CBF/DREB1 responsive elements in the promoters of critical cognate soybean genes.

\section{FIGURE LEGENDS}

Figure 1. Sequence similarity of Arabidopsis and soybean DREB-related genes. Arabidopsis and soybean protein sequences were aligned and calculated to generate a sequence similarity tree using Clustal W2 (http://www.ebi.ac.uk/Tools/msa/clustalw2/). Glyma indicates Glycine max (soybean) $D R E B$ genes, all others are Arabidopsis genes. Soybean nomenclature is from gene model version Wm82.a1.v1 (a convertor tool to convert to newest nomenclature, Wm82.a2.v1, is available at http://www.soybase.org; conversions for genes described are also found in Supplemental Figure Table S2). The dotted line separates the branch containing the GmDREB genes most similar to the AtCBF genes. 
Figure 2. Transcript levels of GmDREB1A;1 \& GmDREB1A;2 (Glyma09g27180 \& Glyma16g32330, respectively) and SCOF-1 (Glyma17g35430) in response to cold stress (c.v. Young) normalized by Actin11 expression. Open circles indicates transcript levels in soybean leaves, closed squares in soybean stems. Fold changes shown are relative to 0 time. Biological replications (1 and 2) represent RNA isolation performed on different experimental plants at different times (at least 4 plants per replicate).

Figure 3. Changes in transcript levels of $G m D R E B$ genes in response to cold stress. RNASEQ analysis was performed in triplicate at all time points (log 10 transcript reads). Each replicate was composed of 6 plants. Standard deviation is shown. Black bar, grey and striped bar indicate 0, 1 and 24 hours of cold stress, respectively. Adjusted p-value $<0.001$ indicated by asterisks are significantly different reads compared to non-cold samples (0 hour). GmDREB1/CBF is the subset of DREB1s that are most similar to the AtCBFs.

Figure 4. Changes in transcript levels of soybean dehydrins in response to cold stress. RNASEQ analysis was performed in triplicate at all time points (log10 scale for transcript reads). Black bar, grey and striped bar indicate 0, 1, and 24 hours of cold stress, respectively. Adjusted p-value $<0.001$ indicated by asterisks are significantly different reads compared to non-cold samples (0 hour). 
Figure 5. Transcript levels of AtCOR47, AtRD29a, and AtADH1 in Arabidopsis transgenic plants (4 weeks old). CBF regulated genes (AtCOR47, AtRD29a and AtADH1) in cold-treated wild-type and in non-cold treated transgenic Arabidopsis expressing GmCBF-like DREBs. Fold-change of transcript accumulation, determined by RT-qPCR, are compared to transcript levels in the 0 hour (no cold) wild-type plants. Expression of sufficient levels of $G m D R E B 1 s$ in the transgenic lines show accumulation of AtCOR47 and AtRD29a transcript without cold stress treatment, similar to cold accumulations due to AtCBF regulation in response to cold. Check mark $(\sqrt{ })$ indicates those lines showing a dwarf phenotype.

Figure 6. Arabidopsis transgenic plants constitutively expressing GmDREB1 genes. Three week old transgenic plants were photographed. Transcript levels of each GmDREB1 in four week old seedlings were absolute quantified to show the number of transcripts in $6.25 \mathrm{ng}$ total RNA by RT-qPCR analysis.

Figure 7. The transgenic plants expressing a sufficient level of GmDREB1 have freezing tolerance. Month old transgenic plants were treated in $-4.5^{\circ} \mathrm{C}$ for 24 hours in dark followed by $4{ }^{\circ} \mathrm{C}$ for 24 hours in the dark and then returned to normal growth conditions for two days for recovery. The photographs were taken after recovery. 
Figure 8. Transgenic Arabidopsis expressing GmDREB1 genes show enhanced freezing tolerance as determined by electrolyte leakage.

Table 1. Transgenic expression of GmDREB1 genes in Arabidopsis confers enhanced freezing tolerance. Freezing treatment was at $-4.5^{\circ} \mathrm{C}$ for 24 hours on 5 week-old plants followed by $4^{\circ} \mathrm{C}$ for 24 hours in the dark and then returned to normal growth conditions for two days for recovery. The damage scale was scored after three days of the recovery, based on area and extent of damage on leaves (Figure S4). A higher number indicates greater damage.

\section{Supplemental Figures and Tables}

Figure S1. Changes in transcript levels of SCOF-1 genes in response to cold stress. RNASEQ analysis was performed in triplicate at all time points (log2 scale); each replicate was composed of 6 plants. Standard deviation is shown. Black bar, white and striped bar indicate 0, 1 and 24 hours of cold stress, respectively. Asterisk indicates significant difference (adjusted P-value $<0.001$ ) as compared to 0 hour cold.

Figure S2. (A) Transcript levels of soybean homologs to cold responsive genes for two dehydrins (KS-dehydrin and GmERD14), alcohol dehydrogenase (ADH), RAP2.1, and LEA14. Cold treatment was initiated with 10 day-old seedlings at ZT-4 h (4h 
after dawn, $18 \mathrm{~h}$ light / $6 \mathrm{~h}$ dark). Samples were harvested at 0h, 1h, $4 \mathrm{~h}$ and $48 \mathrm{~h}$ after initiation of cold $\left(4^{\circ} \mathrm{C}\right)$ treatment. Total RNA was isolated, treated with DNase, reverse transcribed with oligo-dT as primer, then analyzed by semi-quantitative RTPCR. Samples previously used for dehydrin transcript analysis (KS-dehydrin: Glyma17g24193.1 and GmERD14: Glyma04g01130.1; Figure 7, Yamasaki et al., 2013) were compared to $A D H-1, R A P 2.1$, and LEA14. (B) The locations of predicted core elements, ABRE and CRT/DRE, in the promoter regions are shown.

Figure S3. Accumulation of AtCBF3 transcript in Arabidopsis in response to cold stress. Cold treatment $\left(4^{\circ} \mathrm{C}\right)$ on Col-2 (wild-type) started at ZT-4h (18 hours day/ 6 hours night). Fold-change ( $\left.\log _{10}\right)$ of transcript accumulation was calculated by comparison to transcript levels in the 0 hour (no cold) wild-type plants.

Supplement Figure S4. Damage scale (0 to 6) for intact Arabidopsis plants following freezing treatment.

Supplement Figure S5. The reduced flowering structure of Arabidopsis expressing GmDREB1s is partially complemented by GA3. Transgenic plants containing GmDREB1 gene were treated with and without GA3 treatment $\left(10^{-4} \mathrm{M}\right)$ once a week starting at 10 days old. A (non-transformed wild-type, Col-II), B (1302C-1-4: 
35S::GFP), C (09B-1-6: 35S::GmDREB1A;1), D (09B-2-2: 35S::GmDREB1A;1), E

(09C-6-2: 35S::GmDREB1A;1), F (16A-1-5: 35S::GmDREB1A;2), G (16A-5-4:

35S::GmDREB1A;2), H (20B-1-5: 35S::GmDREB1B;1), I (20C-4-3:

35S::GmDREB1B;1), J (10A-5-1: 35S::GmDREB1B;2), K (10A-4-1:

35S::GmDREB1B;2). Photographs were taken at 6 weeks.

Supplement Figure S6. The correlation between absolute levels of transgenic GmDREB1s and relative levels of downstream transcripts; AtCOR47, AtRD29a, and AtADH1. Each data point represents a distinct transformant with different expression levels of the GmDREB1. Transcript amount of GmDREB1s are shown as $\log _{10}$.

Table S1. List of primers for Real Time-qPCR analysis, creation of DREB1 constructs and semi-qPCR analysis.

ACKNOWLEDGEMENTS: This work was partially supported by a grant awarded by the United Soybean Board (USB) Project Grant \# 0238 to SKR. We also thank Dr. Tommy Carter (USDA-ARS, North Carolina State University, Raleigh, NC) for providing Glycine max “ Young”, PI508266, and the USDA-ARS National Plant Germplasm system for providing Glycine max, “Williams 82” (PI518671). We also thank Jennifer Robison (IUPUI) for reviewing this article and providing insightful comments. 


\section{References}

[1] T.M. Cheesbrough, Decreased growth temperature increases soybean stearoylacyl carrier protein desaturase activity, Plant Physiol, 93 (1990) 555-559.

[2] M.Z. Nouri, M. Toorchi, H. Komatsu, Proteomics approach for identifying abiotic stress responsive proteins in soybean, InTech, 2011.

[3] W.J. Bramlage, A.C. Leopold, D.J. Parrish, Chilling stress to soybeans during imbibition, Plant Physiology, 61 (1978) 525-529.

[4] H.A. Kratsch, R.R. Wise, The Ultrastructure of chilling stress, Plant, Cell and Environment, 23 (2000) 337-350.

[5] D.A. Littlejohns, J.W. Tanner, Preliminary studies on teh cold tolerance of soybean seedlings, Can J Plt Sci, 56 (1976) 371-375.

[6] A. Soldati, E.R. Keller, Soybeans adapted to cooler regions, Westview Press, Boulder, USA, 1985.

[7] S.J. Gilmour, R.K. Hajela, M.F. Thomashow, Cold Acclimation in Arabidopsis thaliana, Plant physiology, 87 (1988) 745-750.

[8] M. Uemura, R.A. Joseph, P.L. Steponkus, Cold Acclimation of Arabidopsis thaliana (Effect on Plasma Membrane Lipid Composition and Freeze-Induced Lesions), Plant physiology, 109 (1995) 15-30.

[9] S. Fowler, M.F. Thomashow, Arabidopsis Transcriptome Profiling Indicates That Multiple Regulatory Pathways Are Activated during Cold Acclimation in Addition to the CBF Cold Response Pathway, The Plant Cell Online, 14 (2002) 1675-1690. [10] S. Park, C.M. Lee, C.J. Doherty, S.J. Gilmour, Y. Kim, M.F. Thomashow, Regulation of the Arabidopsis CBF regulon by a complex low-temperature regulatory network, Plant J., 82 (2015) 193-207.

[11] M.F. Thomashow, Molecular basis of plant cold acclimation: insights gained from studying the CBF cold response pathway, Plant Physiol, 154 (2010) 571-577. [12] Q. Liu, M. Kasuga, Y. Sakuma, H. Abe, S. Miura, K. Yamaguchi-Shinozaki, K. Shinozaki, Two transcription factors, DREB1 and DREB2, with an EREBP/AP2 DNA binding domain separate two cellular signal transduction pathways in droughtand low-temperature-responsive gene expression, respectively, in Arabidopsis, Plant Cell, 10 (1998) 1391-1406.

[13] J. Medina, M. Bargues, J. Terol, M. Perez-Alonso, J. Salinas, The Arabidopsis CBF gene family is composed of three genes encoding AP2 domain-containing proteins whose expression Is regulated by low temperature but not by abscisic acid or dehydration, Plant physiology, 119 (1999) 463-470.

[14] V. Haake, D. Cook, J.L. Riechmann, O. Pineda, M.F. Thomashow, J.Z. Zhang, Transcription factor CBF4 is a regulator of drought adaptation in Arabidopsis, Plant physiology, 130 (2002) 639-648.

[15] C.J. Lim, J.E. Hwang, H. Chen, J.K. Hong, K.A. Yang, M.S. Choi, K.O. Lee, W.S. Chung, S.Y. Lee, C.O. Lim, Over-expression of the Arabidopsis DRE/CRTbinding transcription factor DREB2C enhances thermotolerance, Biochem Biophys Res Commun, 362 (2007) 431-436.

[16] D. Hao, K. Yamasaki, A. Sarai, M. Ohme-Takagi, Determinants in the sequence specific binding of two plant transcription factors, CBF1 and NtERF2, to the DRE and GCC motifs., Biochemistry, 41 (2002) 4202-4208. 
[17] K. Maruyama, M. Takeda, S. Kidokoro, K. Yamada, Y. Sakuma, K. Urano, M. Fujita, K. Yoshiwara, S. Matsukura, Y. Morishita, R. Sasaki, H. Suzuki, K. Saito, D. Shibata, K. Shinozaki, K. Yamaguchi-Shinozaki, Metabolic pathways involved in cold acclimation identified by integrated analysis of metabolites and transcripts regulated by DREB1A and DREB2A, Plant physiology, 150 (2009) 1972-1980. [18] National Center for Biotechnology Information (NCBI) GenBank.

[19] K. Maruyama, D. Todaka, J. Mizoi, T. Yoshida, S. Kidokoro, S. Matsukura, H. Takasaki, T. Sakurai, Y.Y. Yamamoto, K. Yoshiwara, M. Kojima, H. Sakakibara, K. Shinozaki, K. Yamaguchi-Shinozaki, Identification of cis-acting promoter elements in cold- and dehydration-induced transcriptional pathways in Arabidopsis, rice, and soybean., DNA Res, 19 (2012) 37-49.

[20] K. Maruyama, Y. Sakuma, M. Kasuga, Y. Ito, M. Seki, H. Goda, Y. Shimada, S. Yoshida, K. Shinozaki, K. Yamaguchi-Shinozaki, Identification of cold-inducible downstream genes of the Arabidopsis DREB1A/CBF3 transcriptional factor using two microarray systems, Plant J., 38 (2004) 982 - 993.

[21] K. Maruyama, M. Takeda, S. Kidokoro, K. Yamada, Y. Sakuma, K. Urano, M. Fujita, K. Yoshiwara, S. Matsukura, Y. Morishita, R. Sasaki, H. Suzuki, K. Saito, D. Shibata, K. Shinozaki, K. Yamaguchi-Shinozaki, Metabolic pathways involved in cold acclimation identified by integrated analysis of metabolites and transcripts regulated by DREB1A and DREB2A, in: Plant Physiol, 2009, pp. 1972-1980. [22] Y. Sakuma, K. Maruyama, Y. Osakabe, F. Qin, M. Seki, K. Shinozaki, K. Yamaguchi-Shinozaki, Functional analysis of an Arabidopsis transcription factor, DREB2A, involved in drought-responsive gene expression, Plant Cell, 18 (2006) 1292-1309.

[23] K.R. Jaglo-Ottosen, S.J. Gilmour, D.G. Zarka, O. Schabenberger, M.F. Thomashow, Arabidopsis CBF1 overexpression induces COR genes and enhances freezing tolerance, Science, 280 (1998) 104-106.

[24] S.J. Gilmour, S.G. Fowler, M.F. Thomashow, Arabidopsis transcriptional activators CBF1, CBF2, and CBF3 have matching functional activities., Plant Mol Biol, 54 (2004) 767-781.

[25] S.J. Gilmour, A.M. Sebolt, M.P. Salazar, J.D. Everard, M.F. Thomashow, Overexpression of the Arabidopsis CBF3 transcriptional activator mimics multiple biochemical changes associated with cold acclimation, Plant physiology, 124 (2000) 1854-1865.

[26] J. Mizoi, T. Ohori, T. Moriwaki, S. Kidokoro, D. Todaka, K. Maruyama, K. Kusakabe, Y. Osakabe, K. Shinozaki, K. Yamaguchi-Shinozaki, GmDREB2A;2, a canonical DEHYDRATION-RESPONSIVE ELEMENT-BINDING PROTEIN2-type transcription factor in soybean, is posttranslationally regulated and mediates dehydration-responsive element-dependent gene expression., Plant Physiol, 161 (2013) 346-361.

[27] T.-H. Hsieh, J.-T. Lee, P.-T. Yang, L.-H. Chiu, Y.-y. Charng, Y.-C. Wang, M.-T. Chan, Heterology Expression of the ArabidopsisC-Repeat/Dehydration Response Element Binding Factor 1 Gene Confers Elevated Tolerance to Chilling and Oxidative Stresses in Transgenic Tomato, Plant Physiology, 129 (2002) 1086-1094. [28] T.H. Hsieh, J.T. Lee, P.T. Yang, L.H. Chiu, Y.Y. Charng, Y.C. Wang, M.T. Chan, Heterology expression of the Arabidopsis C-repeat/dehydration response 
element binding factor 1 gene confers elevated tolerance to chilling and oxidative stresses in transgenic tomato., Plant physiology, 129 (2002) 1086-1094.

[29] Y.J. Zhang, J.S. Yang, S.J. Guo, J.J. Meng, Y.L. Zhang, S.B. Wan, Q.W. He, X.G. Li, Over-expression of the Arabidopsis CBF1 gene improves resistance of tomato leaves to low temperature under low irradiance., Plant Biol, 13 (2011) 362367.

[30] X. Zhang, S.G. Fowler, H. Cheng, Y. Lou, S.Y. Rhee, E.J. Stockinger, M.F. Thomashow, Freezing-sensitive tomato has a functional CBF cold response pathway, but a CBF regulon that differs from that of freezing-tolerant Arabidopsis, Plant J., 39 (2004) 905-919.

[31] J.C. Kim, S.H. Lee, Y.H. Cheong, C.M. Yoo, S.I. Lee, H.J. Chun, D.J. Yun, J.C. Hong, S.Y. Lee, C.O. Lim, M.J. Cho, A novel cold-inducible zinc finger protein from soybean, SCOF-1, enhances cold tolerance in transgenic plants, Plant J, 25 (2001) 247-259.

[32] M. Chen, Q.Y. Wang, X.G. Cheng, Z.S. Xu, L.C. Li, X.G. Ye, L.Q. Xia, Y.Z. Ma, GmDREB2, a soybean DRE-binding transcription factor, conferred drought and high-salt tolerance in transgenic plants, Biochem Biophys Res Commun, 353 (2007) 299-305.

[33] Y.H. Kim, M.D. Kim, S.C. Park, K.S. Yang, J.C. Jeong, H.S. Lee, S.S. Kwak, SCOF-1-expressing transgenic sweetpotato plants show enhanced tolerance to lowtemperature stress., Plant Physiol Biochem, 49 (2011) 1436-1441.

[34] D.J. Hume, A.K. Jackson, Frost tolerance in soybean, Crop Science, 21 (1981) 689-692.

[35] Y. Yamasaki, G. Koehler, B.J. Blacklock, S.K. Randall, Dehydrin expression in soybean, Plant Physiol Biochem, 70 (2013) 213-220.

[36] S. Komatsu, T. Deschamps, S. Hiraga, M. Kato, M. Chiba, A. Hashiguchi, M. Tougou, S. Shimamura, H. Yasue, Characterization of a novel flooding stressresponsive alcohol dehydrogenase expressed in soybean roots, Plant Mol Biol, 77 (2011) 309-322.

[37] S. Kidokoro, K. Watanabe, T. Ohori, T. Moriwaki, K. Maruyama, J. Mizoi, N. Myint Phyu Sin Htwe, Y. Fujita, S. Sekita, K. Shinozaki, K. Yamaguchi-Shinozaki, Soybean DREB1/CBF-type transcription factors function in heat and drought as well as cold stress-responsive gene expression, Plant J., 81 (2015) 505-518.

[38] M. Martinez-Trujillo, V. Limones-Briones, J.L. Cabrera-Ponce, L. HerreraEstrella, Improving transformation efficiency ofArabidopsis thaliana by modifying the floral dip method, Plant Molecular Biology Reporter, 22 (2004) 63-70.

[39] S.J. Harrison, E.K. Mott, K. Parsley, S. Aspinall, J.C. Gray, A. Cottage, A rapid and robust method of identifying transformed Arabidopsis thaliana seedlings following floral dip transformation, Plant methods, 2 (2006) 19.

[40] Y. Chen, P. Chen, B.G. Reyes, Differential responses of the cultivated and wild species of soybean to dehydration stress, Crop Science, 46 (2006) 2041-2046.

[41] J. Schmutz, S.B. Cannon, J. Schlueter, J. Ma, T. Mitros, W. Nelson, D.L. Hyten, Q. Song, J.J. Thelen, J. Cheng, D. Xu, U. Hellsten, G.D. May, Y. Yu, T. Sakurai, T. Umezawa, M.K. Bhattacharyya, D. Sandhu, B. Valliyodan, E. Lindquist, M. Peto, D. Grant, S. Shu, D. Goodstein, K. Barry, M. Futrell-Griggs, B. Abernathy, J. Du, Z. Tian, L. Zhu, N. Gill, T. Joshi, M. Libault, A. Sethuraman, X.C. Zhang, K. Shinozaki, 
H.T. Nguyen, R.A. Wing, P. Cregan, J. Specht, J. Grimwood, D. Rokhsar, G. Stacey, R.C. Shoemaker, S.A. Jackson, Genome sequence of the palaeopolyploid soybean, Nature, 463 (2010) 178-183.

[42] F. Novillo, J.M. Alonso, J.R. Ecker, J. Salinas, CBF2/DREB1C is a negative regulator of CBF1/DREB1B and CBF3/DREB1A expression and plays a central role in stress tolerance in Arabidopsis, Proc Natl Acad Sci U S A, 101 (2004) 3985-3990. [43] S. Fowler, M.F. Thomashow, Arabidopsis transcriptome profiling indicates that multiple regulatory pathways are activated during cold acclimation in addition to the CBF cold response pathway, Plant Cell, 14 (2002) 1675-1690.

[44] J.C. Pennycooke, H. Cheng, S.M. Roberts, Q. Yang, S.Y. Rhee, E.J. Stockinger, The low temperature-responsive, Solanum CBF1 genes maintain high identity in their upstream regions in a genomic environment undergoing gene duplications, deletions, and rearrangements, Plant Mol Biol, 67 (2008) 483-497.

[45] V. Chinnusamy, M. Ohta, S. Kanrar, B.H. Lee, X. Hong, M. Agarwal, J.K. Zhu, ICE1: a regulator of cold-induced transcriptome and freezing tolerance in Arabidopsis, Genes Dev, 17 (2003) 1043-1054.

[46] C.J. Doherty, H.A. Van Buskirk, S.J. Myers, M.F. Thomashow, Roles for Arabidopsis CAMTA transcription factors in cold-regulated gene expression and freezing tolerance, Plant Cell, 21 (2009) 972-984.

[47] Y. Kim, S. Park, S.J. Gilmour, M.F. Thomashow, Roles of CAMTA transcription factors and salicylic acid in configuring the low-temperature transcriptome and freezing tolerance of Arabidopsis, Plant J., 75 (2013) 364-376. [48] T. Meshi, M. Iwabuchi, Plant transcription factors, Plant Cell Physiol, 36 (1995) 1405-1420.

[49] K. Maruyama, M. Takeda, S. Kidokoro, K. Yamada, Y. Sakuma, K. Urano, M. Fujita, K. Yoshiwara, S. Matsukura, Y. Morishita, R. Sasaki, H. Suzuki, K. Saito, D. Shibata, K. Shinozaki, K. Yamaguchi-Shinozaki, Metabolic Pathways Involved in Cold Acclimation Identified by Integrated Analysis of Metabolites and Transcripts Regulated by DREB1A and DREB2A, Plant Physiology, 150 (2009) 1972-1980. [50] B.-h. Lee, D.A. Henderson, J.-K. Zhu, The Arabidopsis Cold-Responsive Transcriptome and Its Regulation by ICE1, The Plant Cell Online, 17 (2005) 31553175.

[51] K. Maruyama, Y. Sakuma, M. Kasuga, Y. Ito, M. Seki, H. Goda, Y. Shimada, S. Yoshida, K. Shinozaki, K. Yamaguchi-Shinozaki, Identification of cold-inducible downstream genes of the Arabidopsis DREB1A/CBF3 transcriptional factor using two microarray systems, Plant J, 38 (2004) 982-993.

[52] H. Magome, S. Yamaguchi, A. Hanada, Y. Kamiya, K. Oda, dwarf and delayedflowering 1, a novel Arabidopsis mutant deficient in gibberellin biosynthesis because of overexpression of a putative AP2 transcription factor., Plant J, 37 (2004) 720-729. [53] J.A. Jarillo, A. Leyva, J. Salinas, J.M. Martinez-Zapater, Low Temperature Induces the Accumulation of Alcohol Dehydrogenase mRNA in Arabidopsis thaliana, a Chilling-Tolerant Plant, Plant physiology, 101 (1993) 833-837.

[54] G.L. de Bruxelles, W.J. Peacock, E.S. Dennis, R. Dolferus, Abscisic acid induces the alcohol dehydrogenase gene in Arabidopsis, Plant physiology, 111 (1996) 381-391. 
[55] M. Ishitani, L. Xiong, B. Stevenson, J.K. Zhu, Genetic analysis of osmotic and cold stress signal transduction in Arabidopsis: interactions and convergence of abscisic acid-dependent and abscisic acid-independent pathways, Plant Cell, 9 (1997) 1935-1949.

[56] H. Lee, L. Xiong, Z. Gong, M. Ishitani, B. Stevenson, J.K. Zhu, The Arabidopsis HOS1 gene negatively regulates cold signal transduction and encodes a RING finger protein that displays cold-regulated nucleo--cytoplasmic partitioning, Genes Dev, 15 (2001) 912-924.

[57] C.H. Dong, M. Agarwal, Y. Zhang, Q. Xie, J.K. Zhu, The negative regulator of plant cold responses, HOS1, is a RING E3 ligase that mediates the ubiquitination and degradation of ICE1, Proc Natl Acad Sci U S A, 103 (2006) 8281-8286. [58] J.T. Vogel, D.G. Zarka, H.A. Van Buskirk, S.G. Fowler, M.F. Thomashow, Roles of the CBF2 and ZAT12 transcription factors in configuring the low temperature transcriptome of Arabidopsis, Plant J., 41 (2005) 195-211.

[59] M. Momma, S. Kaneko, K. Haraguchi, U. Matsukura, Peptide mapping and assessment of cryoprotective activity of 26/27-kDa dehydrin from soybean seeds, Biosci Biotechnol Biochem, 67 (2003) 1832-1835.

[60] M.S. Whitsitt, R.G. Collins, J.E. Mullet, Modulation of dehydration tolerance in soybean seedlings (dehydrin Mat1 Is induced by dehydration but not by abscisic acid), Plant physiology, 114 (1997) 917-925. 


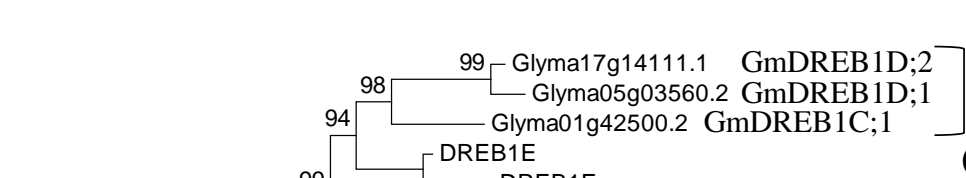

99 99 DREB1F

99 Glyma09g27180.1 GmDREB1A;1 G Glyma16g32330.1 GmDREB1A;

$95[$ G Glyma20g29410.1 GmDREB1B;1

$\mathrm{GmCBF} /$

AtCBF4/DREB1D

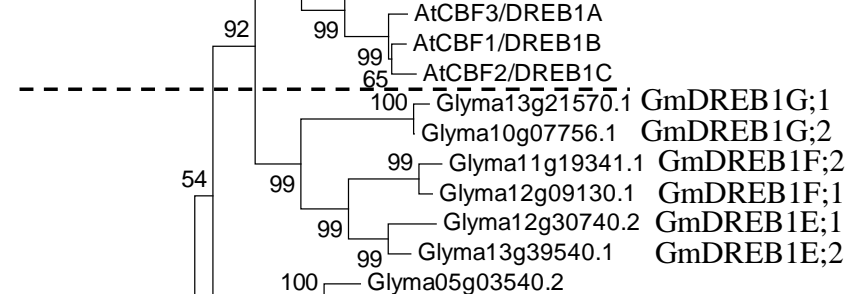

98

G Glyma17g14100.1

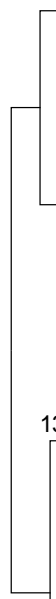

$53 \quad 100$ [ Glyma13g21560.2

$99 \quad$ Glyma19g37670.1

994 Glyma15g02900.1

DREB3

- Glyma02g43240.2 GmABI4;

$99-$ Glyma02g31350.1 GmDREB2G;

99 Glyma03g29530.1 GmDREB2H;1

99 Glyma19g04461.1 GmDREB2I;3
19
DREB2F 2210.1
$\sqrt[19]{\square}$ DREB2G

$23{ }_{86}-$ Glyma06g04490.1

$84 \quad 99 \quad{ }_{9}^{100}{ }^{\text {Glyma14g07620.1 GmDREB2E;1 }}$ Glyma17g37350.2 GmDREB2E;2

$7 5 \longdiv { 9 8 } \quad$ Glyma009030070.2 GmDREB2F;1

DREB2D

$1 2 \quad 3 4 \longdiv { \text { Glyma07g19221.1 GmDREB2D;1 } }$

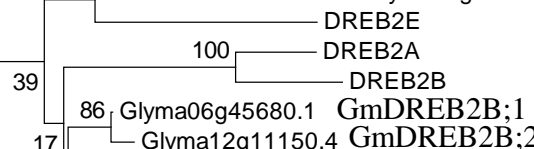
68 Glyma13g38030.1 GmDREB2C;2
$10[$ Glyma12g32400.1 GmDREB2C;1

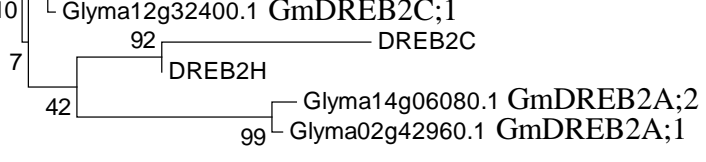



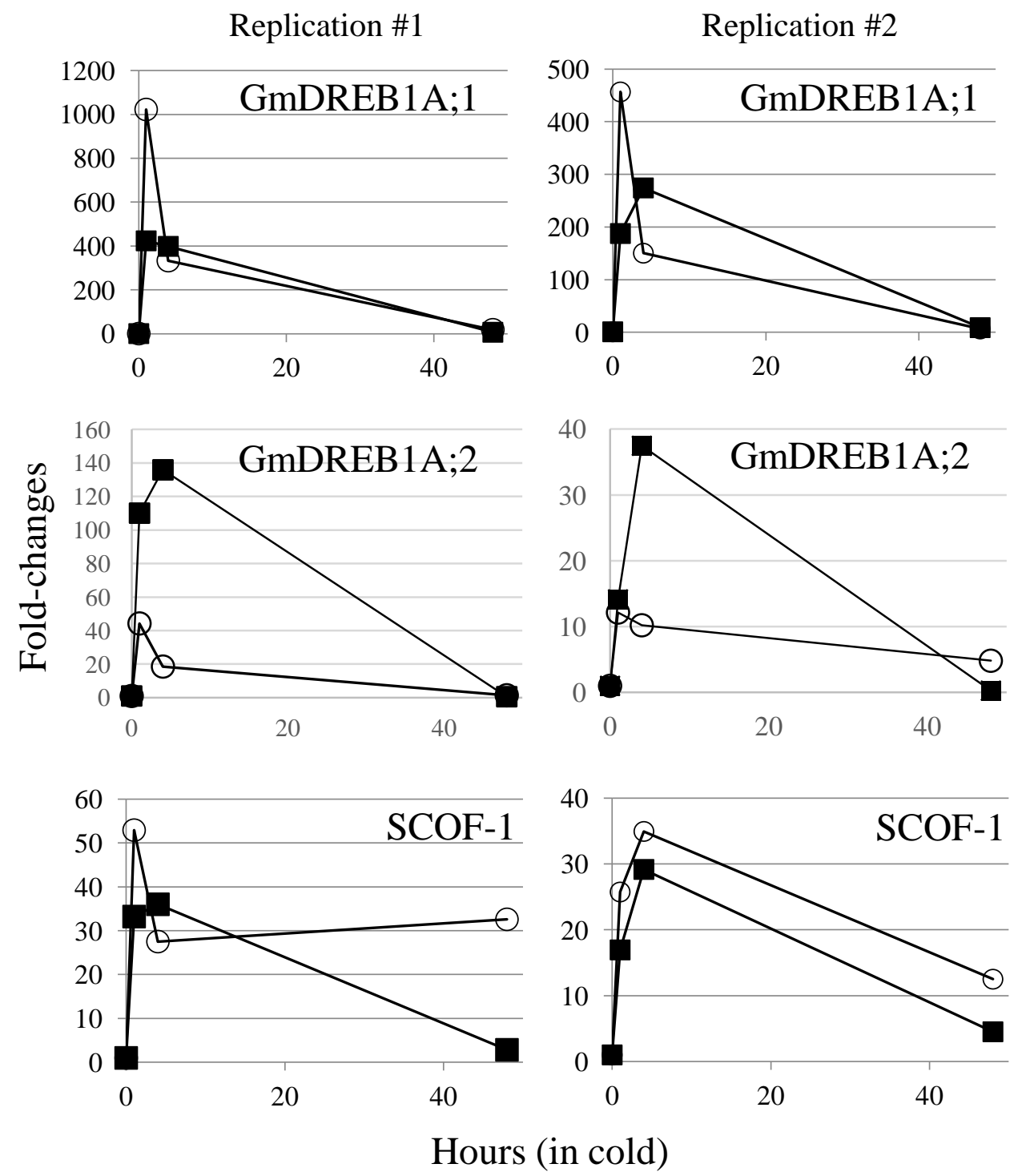


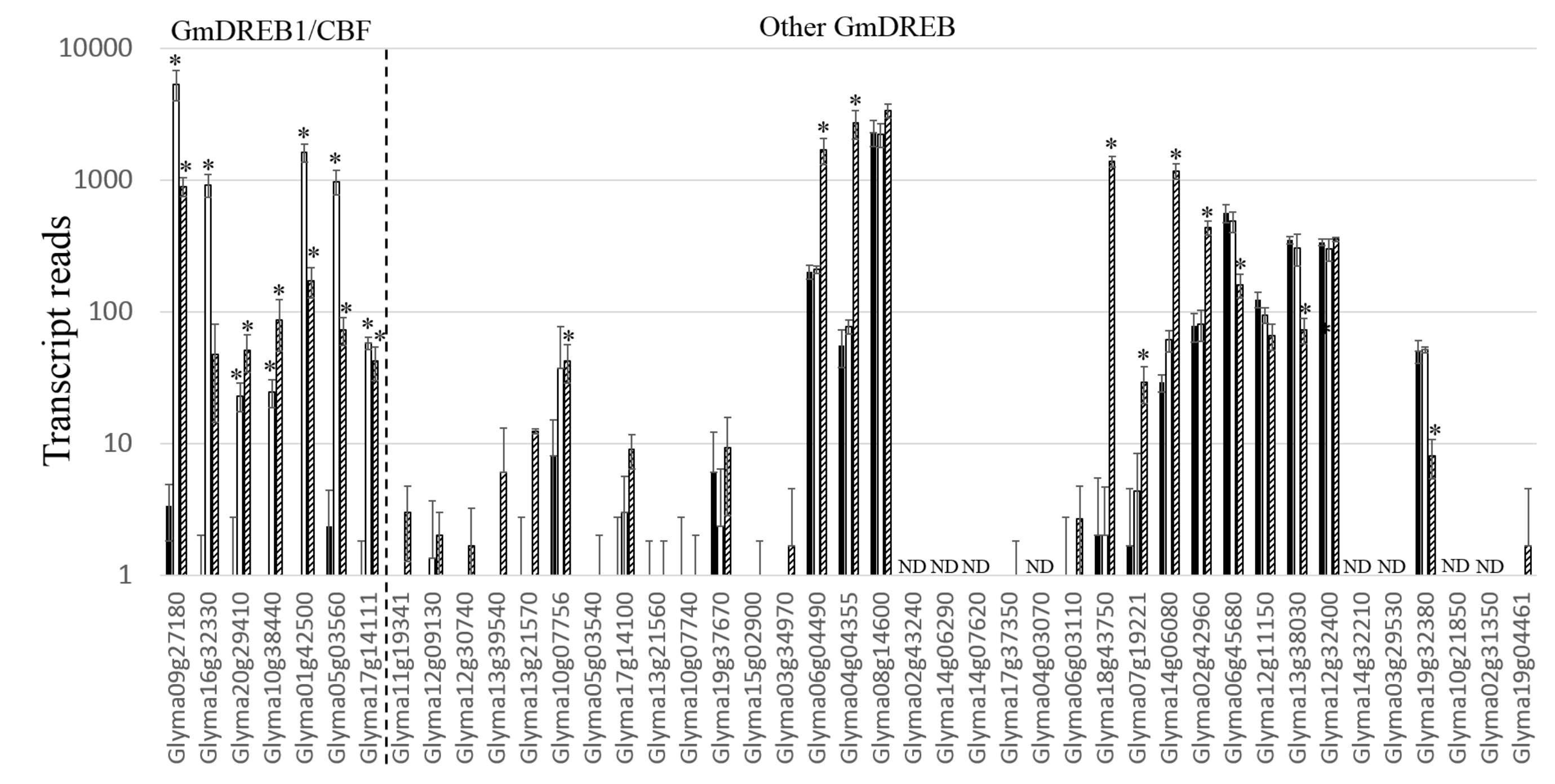




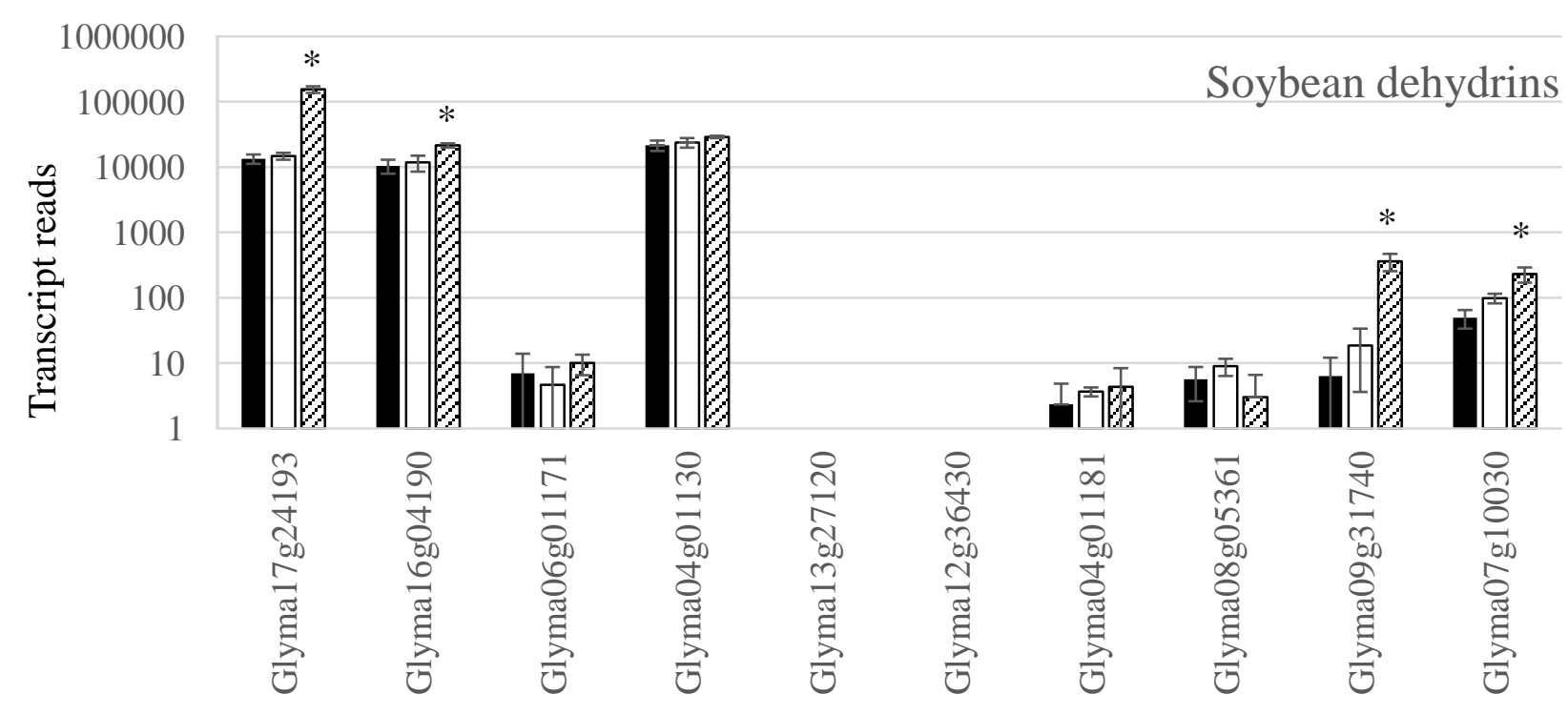




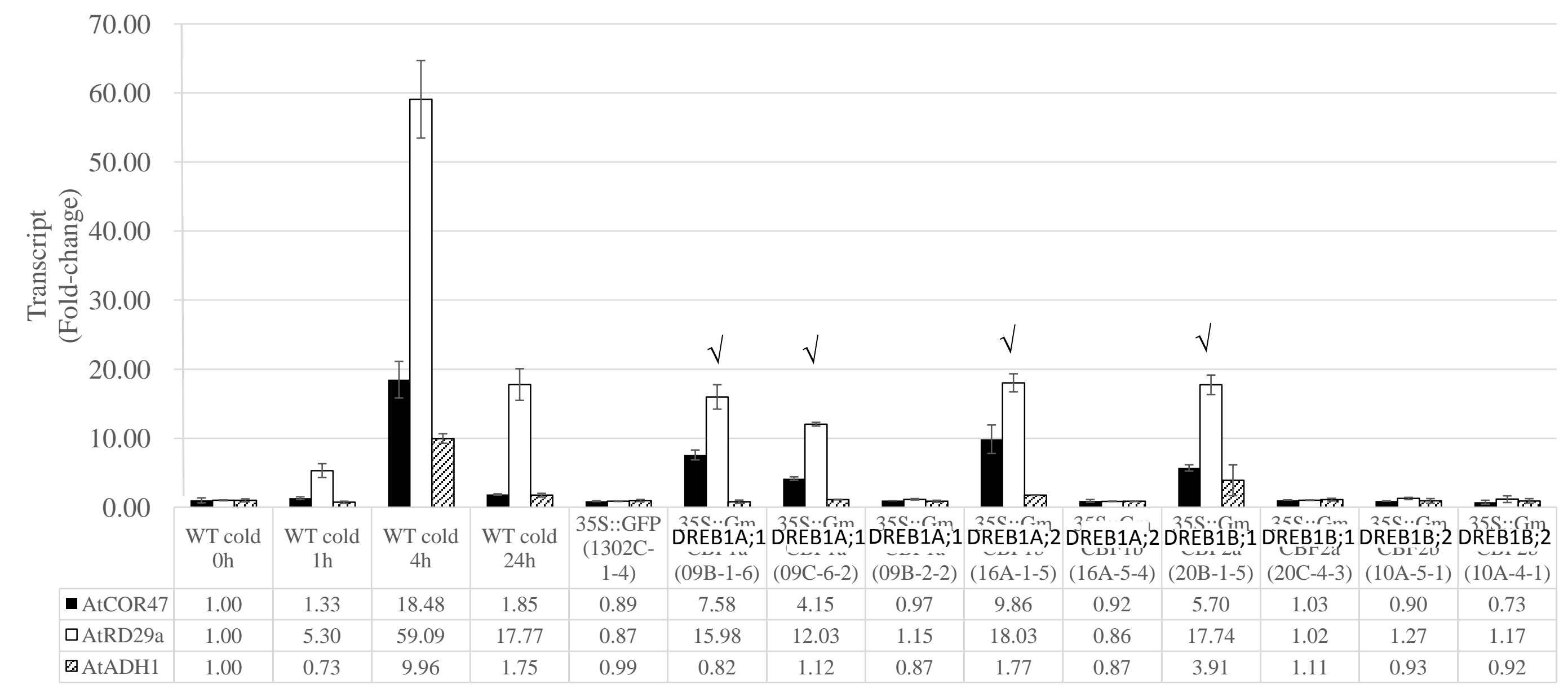

0.82




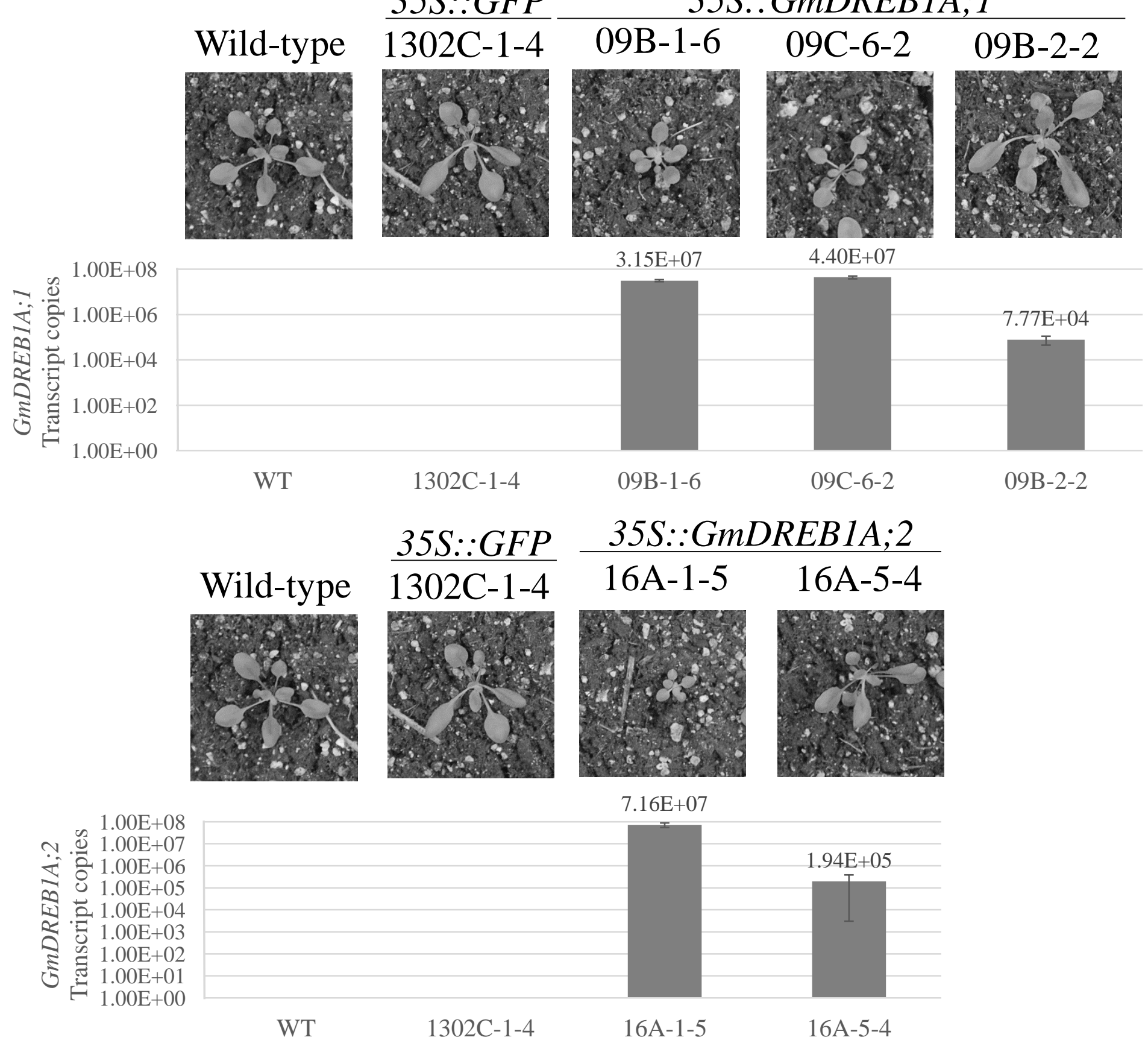




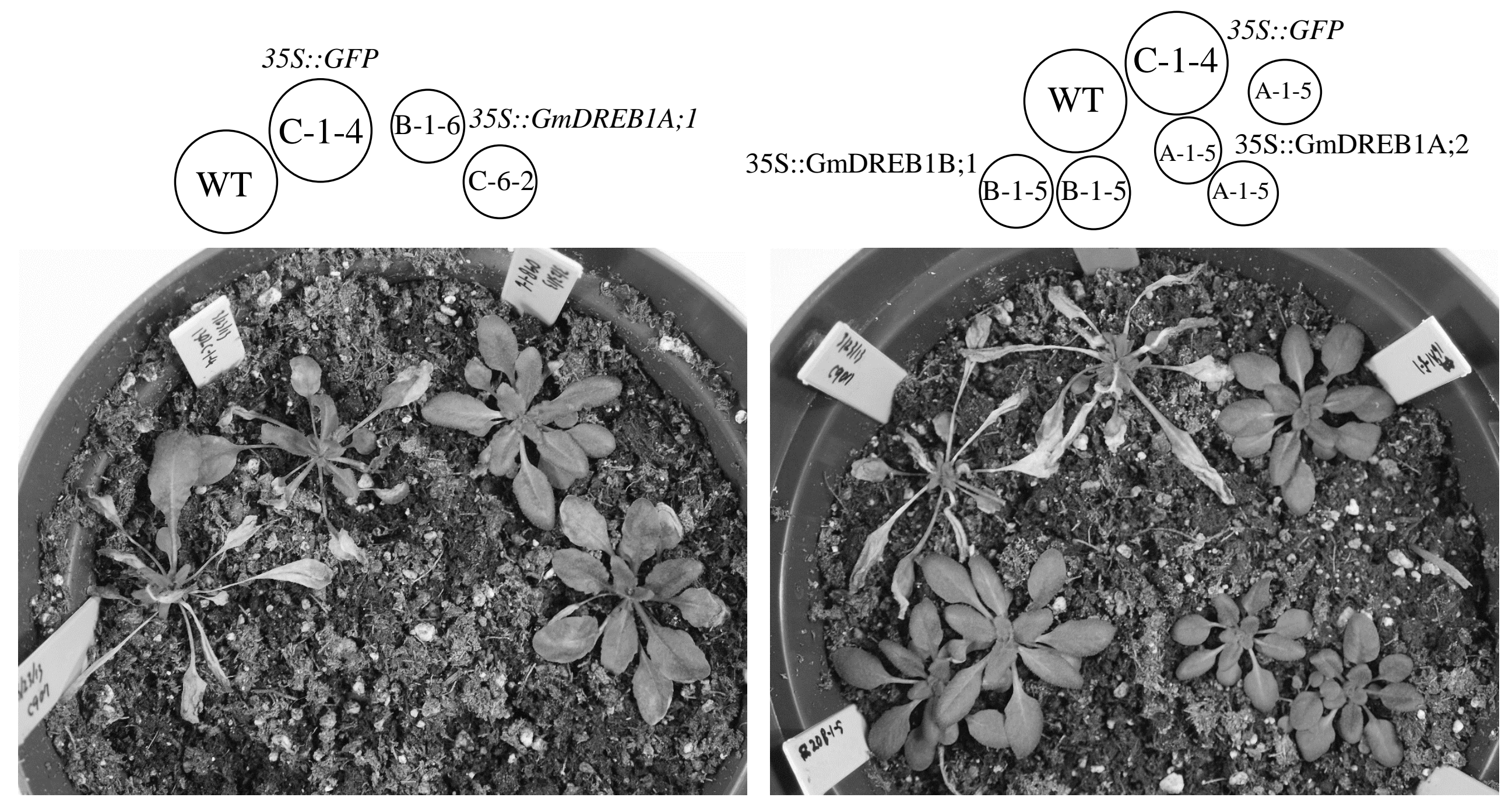




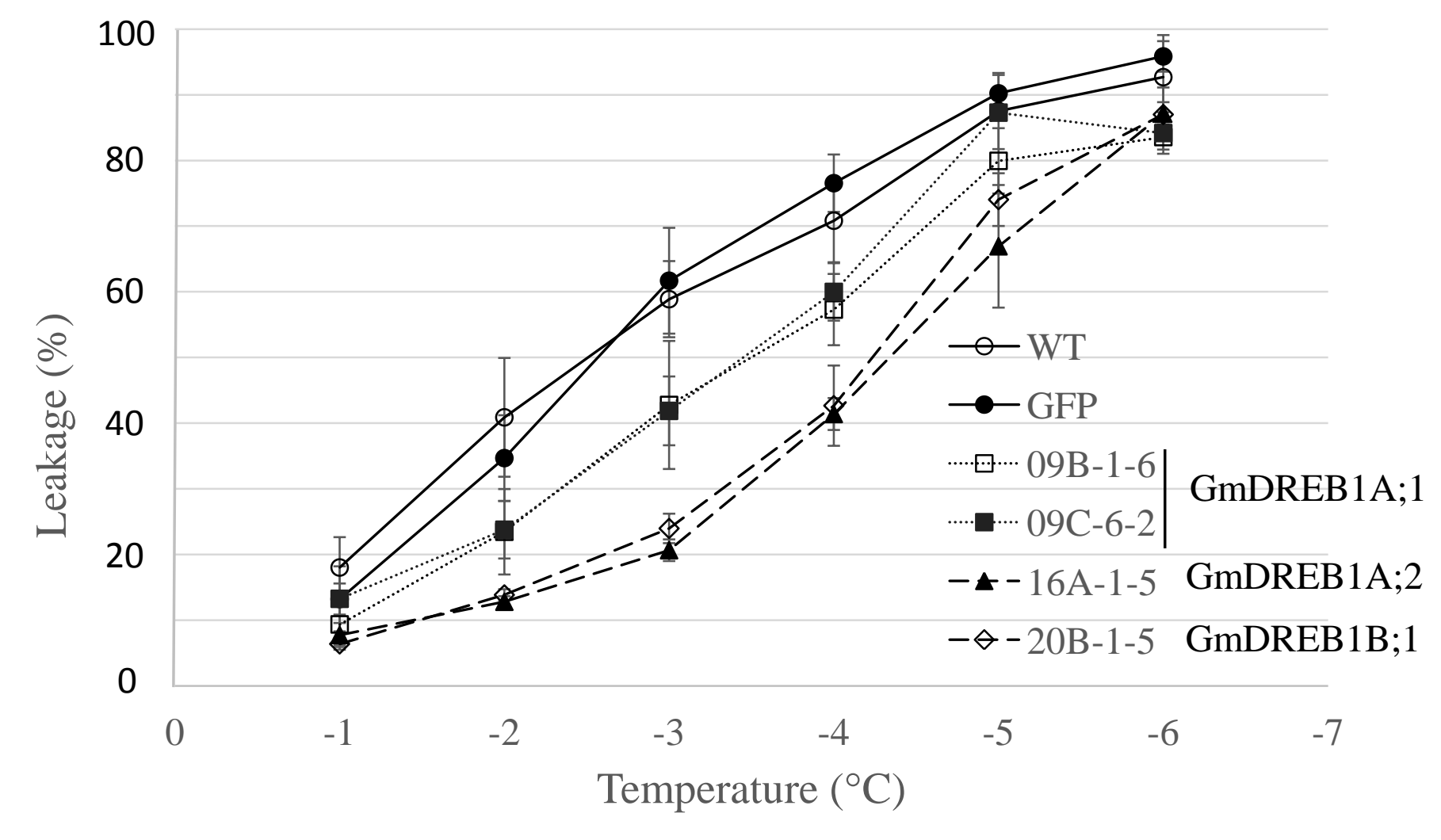




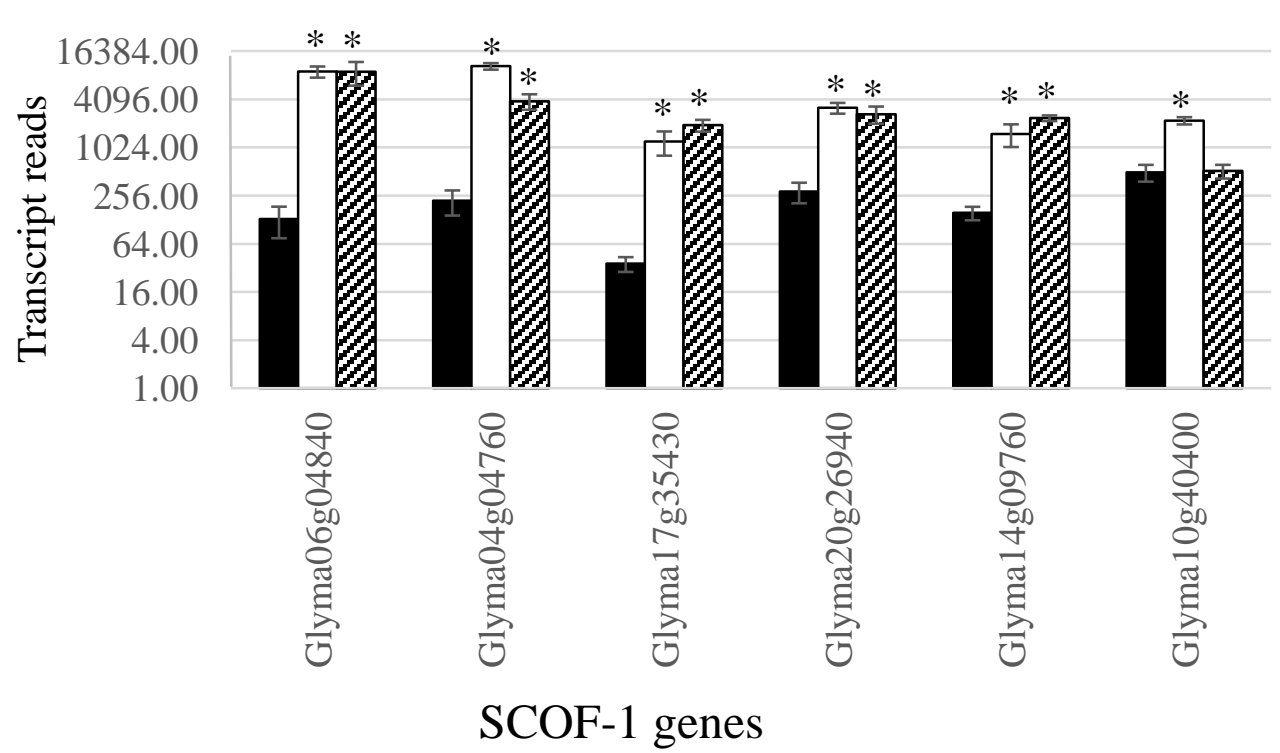


(A) Cold treatment (hours) KS-dehydrin (Glyma17g24193.1) GmERD14 (Glyma04g01130.1)

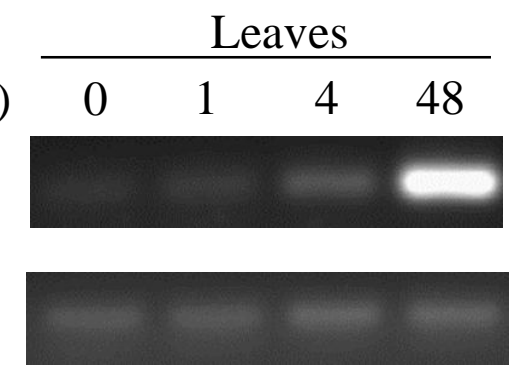

Leaves

Cold treatment (hours)

ADH-1, SoyADH10 (Glyma14g27940.1)

Homolog to AtRAP2.1 (Glyma14g09320.1)

Homolog to AtLEA14 (Glyma14g27940.1)

Soybean Actin
(B)
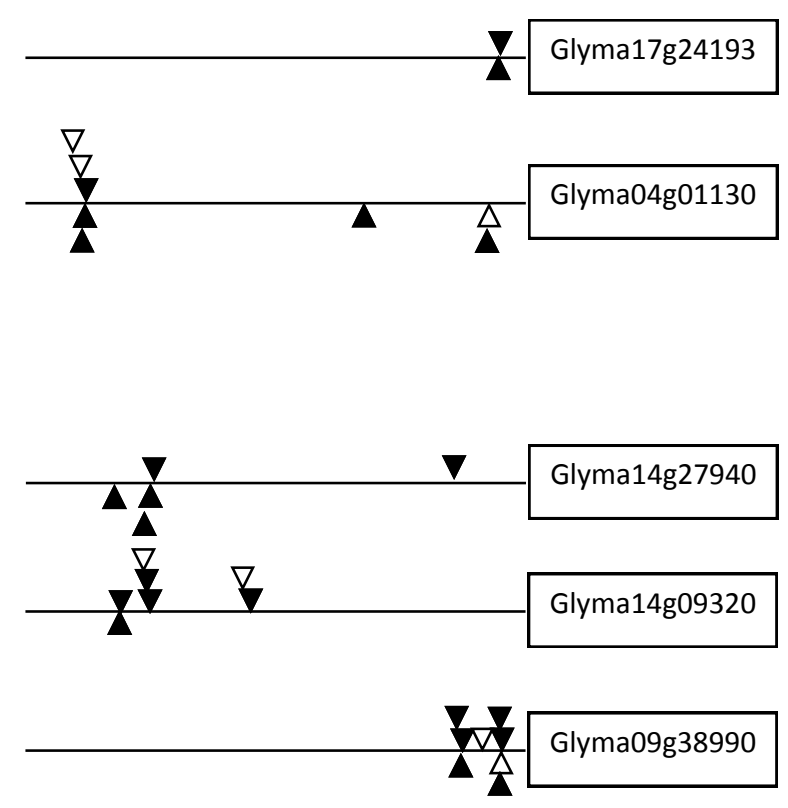

$\longleftrightarrow 500 \mathrm{bp}$

$\nabla$ ABRE

$\triangle A B R E$

$\nabla C R T / D R E$

$\triangle \mathrm{CRT} / \mathrm{DRE}$

5'-ACGTGG/T-3'

5'-A/CCACGT-3'

5'-A/GCCGAC-3'

$5^{\prime}$-GTCGGC/T-3' 


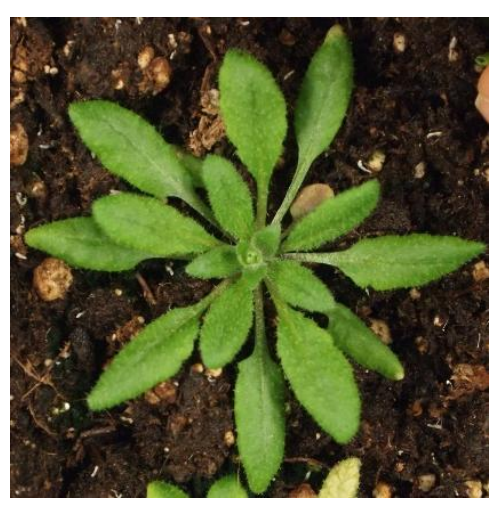

Damage 0

No damage on any leaves

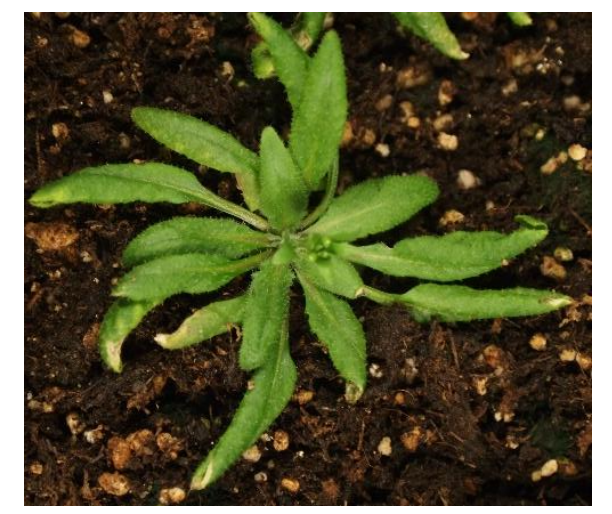

Damage 1

Minor damage (bleached) at the edge of a few leaves

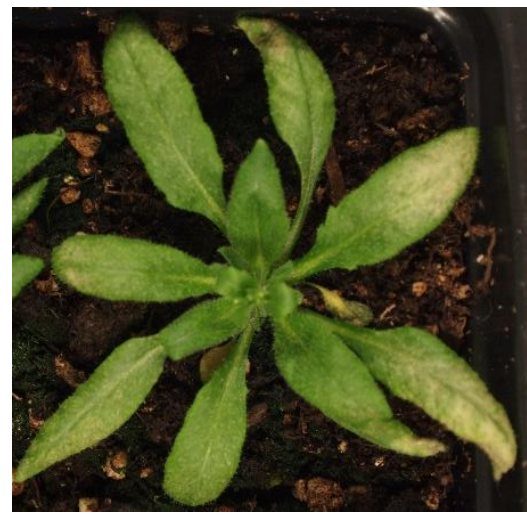

Damage 2

Heavily damaged (wrinkled or leaf form collapsed) on single leaf or minor damage on less than $50 \%$ leaves

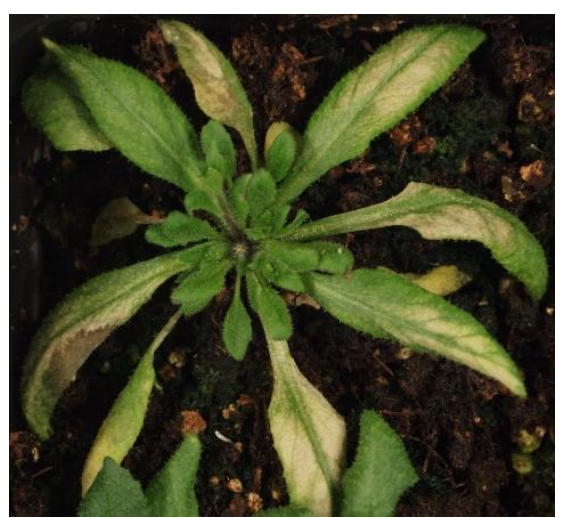

Damage 3

Heavily damaged on multiple leaves or minor damage on more than 50 75\% leaves

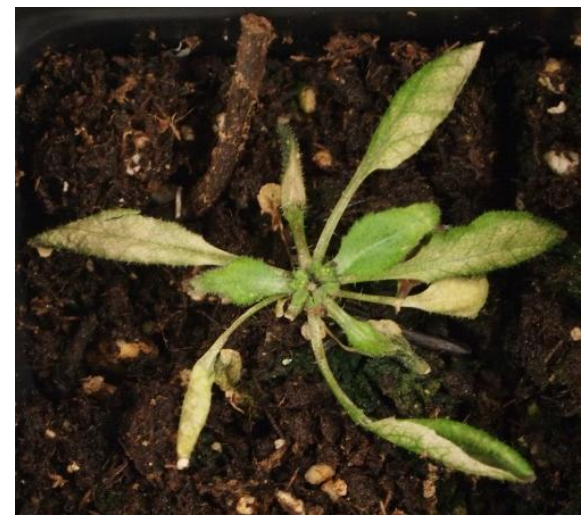

Damage 4

Heavily damaged on $50 \%$ of leaves of a plant and minor damage on more than 50 75\% leaves

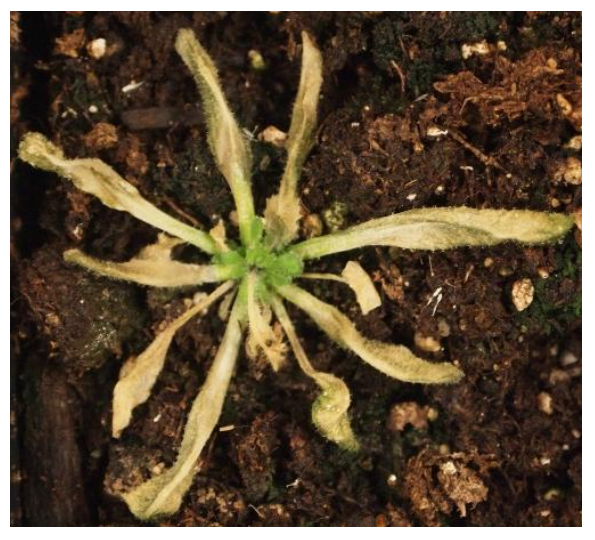

Damage 5

Heavily damaged on most leaves but still remaining green tissue, minor damage (bleached) on more than $75 \%$ leaves

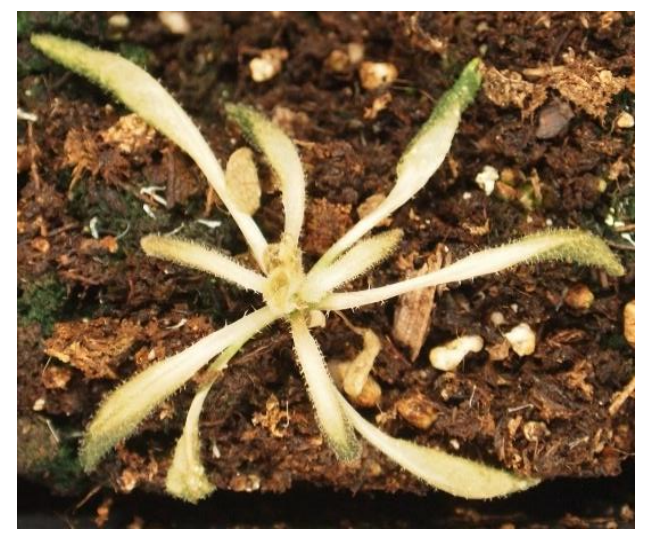

Damage 6

$100 \%$ area of a plant bleached or dehydrated 

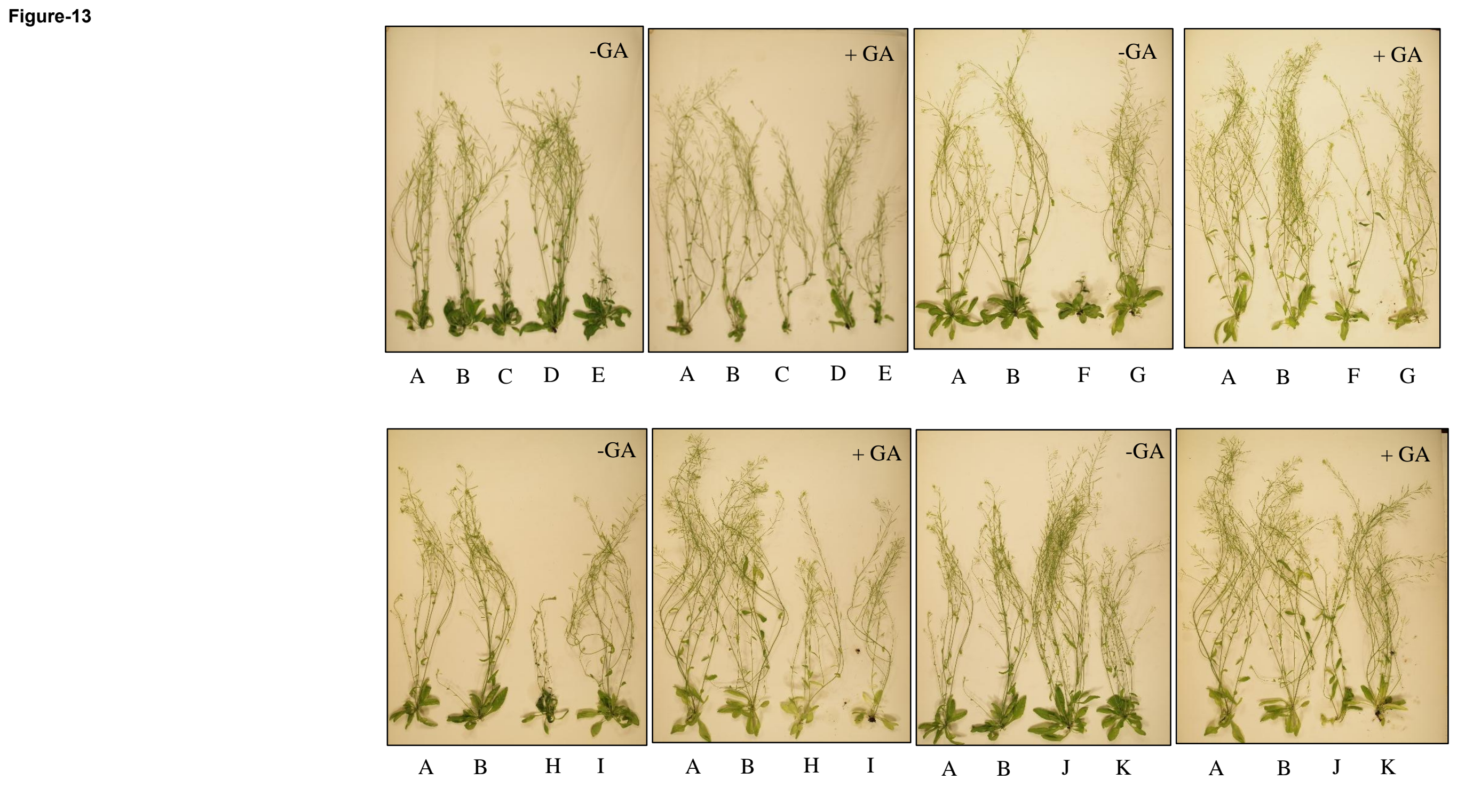

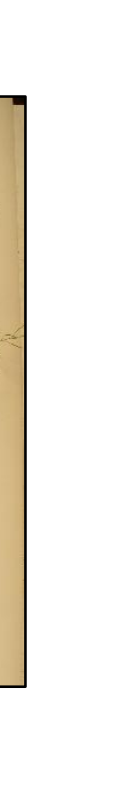

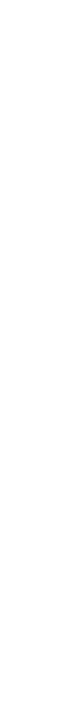


Figure-14
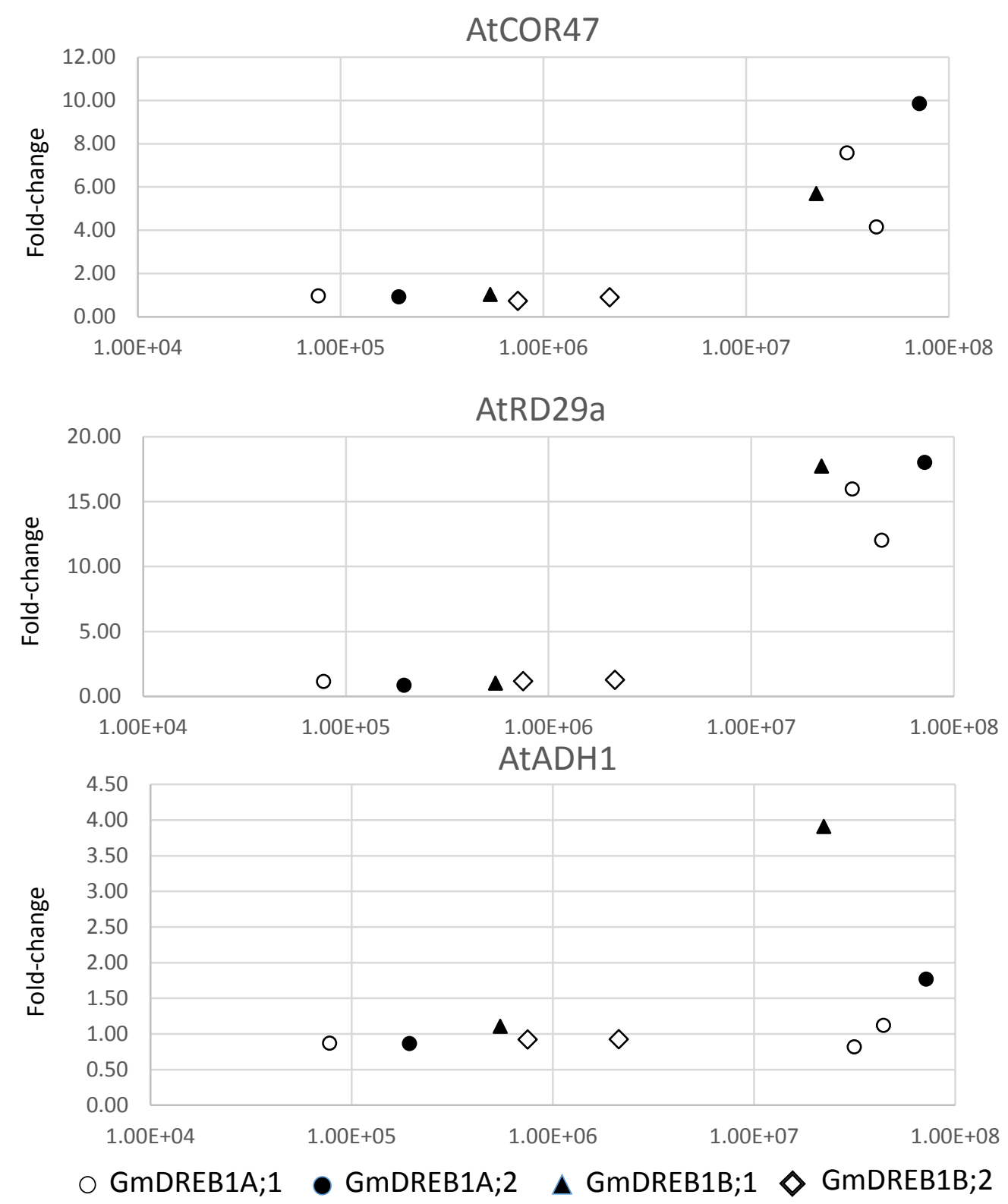

Absolute transcript levels of transgenic soybean DREB1 genes in Arabidopsis (number of transcripts per $6.25 \mathrm{ng}$ RNA) 


\begin{tabular}{|c|c|c|c|c|}
\hline $\begin{array}{c}\text { Transformed } \\
\text { gene }\end{array}$ & Background & $\begin{array}{c}\text { Average } \\
\text { damage }\end{array}$ & $\begin{array}{c}\text { Standard } \\
\text { deviation }\end{array}$ & $\begin{array}{c}\text { Number of } \\
\text { plants (n) }\end{array}$ \\
\hline N/A & WT & 3.17 & 1.38 & 18 \\
\hline $35 S::$ & $1302 \mathrm{C}-1-4$ & 3.06 & 1.52 & 17 \\
GFP & $09 \mathrm{~B}-1-6$ & 0.09 & 0.29 & 23 \\
\cline { 2 - 5 } $35 S::$ & $09 \mathrm{C}-6-2$ & 0.32 & 0.48 & 19 \\
\cline { 2 - 5 } GmDREB1A;1 & $09 \mathrm{~B}-2-2$ & 3.83 & 1.03 & 12 \\
\hline $35 S::$ & $16 \mathrm{~A}-1-5$ & 0.05 & 0.22 & 21 \\
\cline { 2 - 5 } GmDREB1A;2 & $16 \mathrm{~A}-5-4$ & 3.55 & 1.13 & 11 \\
\hline \multirow{3}{*}{$\begin{array}{c}35 S:: \\
\text { GmDREB1B;1 }\end{array}$} & $20 \mathrm{~B}-1-5$ & 0.05 & 0.22 & 20 \\
\cline { 2 - 5 } & $20 \mathrm{C}-4-3$ & 1.78 & 1.09 & 9 \\
\hline $35 S::$ & $10 \mathrm{~A}-5-1$ & 2.00 & 1.26 & 11 \\
\cline { 2 - 5 } GmDREB1B;2 & $10 \mathrm{~A}-4-1$ & 1.83 & 1.80 & 12 \\
\hline
\end{tabular}




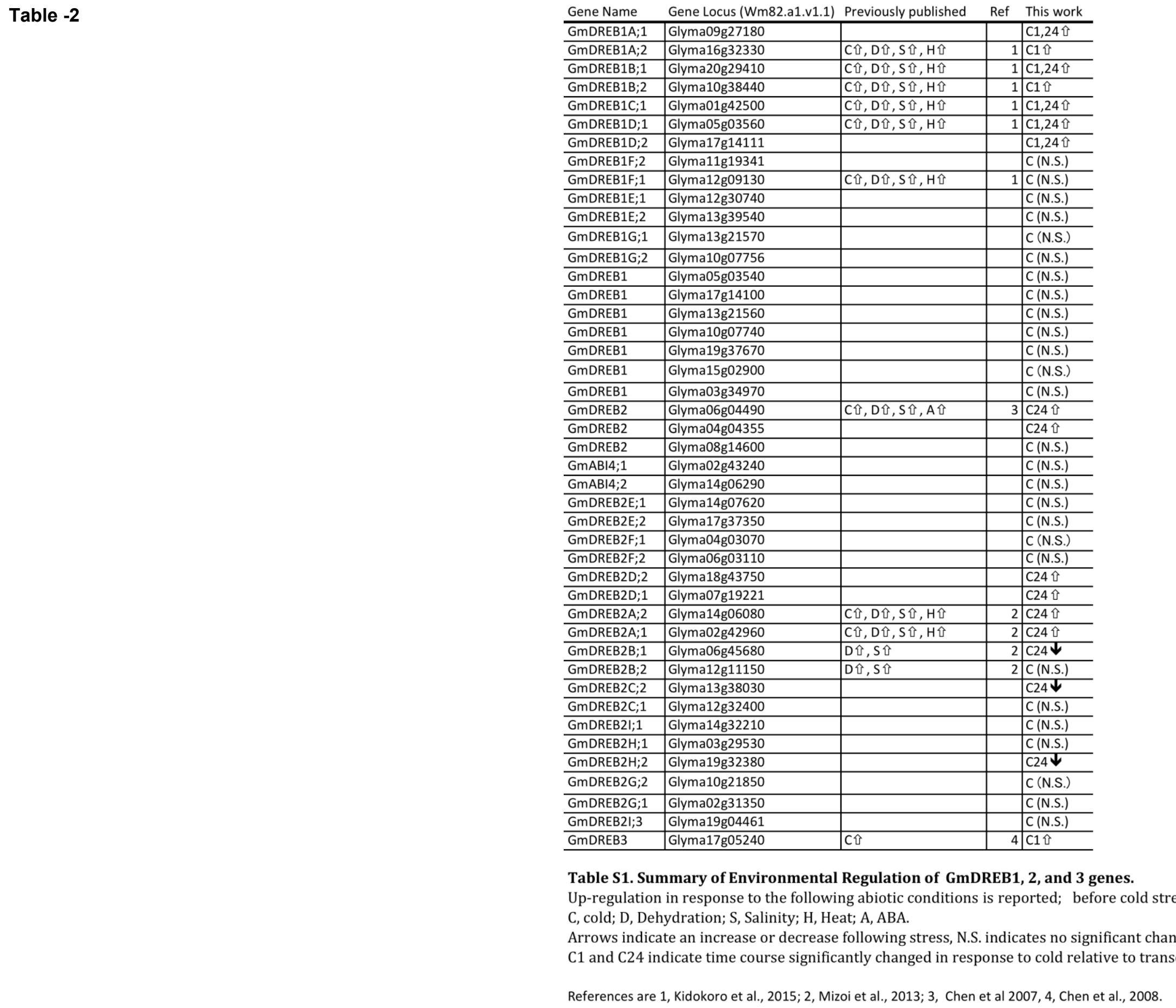

\section{Table S1. Summary of Environmental Regulation of GmDREB1, 2, and 3 genes.}

Up-regulation in response to the following abiotic conditions is reported; before cold stress. C, cold; D, Dehydration; S, Salinity; H, Heat; A, ABA.

Arrows indicate an increase or decrease following stress, N.S. indicates no significant change

C1 and C24 indicate time course significantly changed in response to cold relative to transcript level

References are 1, Kidokoro et al., 2015; 2, Mizoi et al., 2013; 3, Chen et al 2007, 4, Chen et al., 2008 
